\title{
Diffraction of light by ultrasound: Behaviour of the first order and the higher order Bragg maxima
}

\author{
Otohiko Nomoto* and Akiji Ouchi** \\ *Kobayasi Institute of Physical Research, 3-20-41 Higasi-motomati, \\ Kokubunzi, Tokyo, 185 Japan \\ **Department of Mathematics, National Defense Academy, \\ 1-10-20 Hasirimizu, Yokosuka, Kanagawa, 239 Japan
}

(Received 20 May 1980)

\begin{abstract}
Intensity distribution of the light diffraction spectrum produced by progressive ultrasound is calculated by the normal mode theory-extended Brillouin theory-over a wide range of the parameters pertaining to this phenomenon and under condition of the normal incidence and under Bragg incidences of various orders. It is found that both "Bragg maxima" and "Bragg minima" take place for various order spectra according to the ranges of the various parameters pertaining to this phenomenon. The intuitive mechanism for the variation of the spectrum outside of the Raman-Nath region and the true Bragg region is attributable to the mirror-type Bragg reflection anticipated by the ray-optics theory.
\end{abstract}

PACS number: 43.35

\section{INTRODUCTION}

The diffraction of light by ultrasound is known to take very various aspects according to the various experimental parameters pertaining to this phenomenon, even in the simplest two-dimensional model case of the Fraunhofer diffraction of monochromatic light by a plane progressive sinusoidal sound beam of rectangular cross section in a homogeneous isotropic medium, transparent to both light and sound. Indeed, the phenomenon was theoretically anticipated as a Bragg-type Brillouin scattering by L. Brillouin ${ }^{1)}$ in 1922, while the first experimental observations by P. Debye and Sears ${ }^{2)}$ and independently by Lucas and Biquard ${ }^{3)}$ in 1932 disclosed a seemingly quite different aspect phenomenon as compared to the Bragg type diffraction, producing many orders of spectra and observable also under normal incidence. Although the Brillouin's paper included two anticipations, the thermal Brillouin scattering and the diffraction of light by ultrasound, the nature of the anticipated phenomenon for the latter was also of the Brillouin-scattering type satisfying Bragg condition. Today it is known that the ultrasonic light diffraction takes two types in the limiting cases-Bragg type in the high frequency limit and Raman-Nath ${ }^{4}$ type in the low frequency limit. It must be emphasized, however, that there is a wide range of parameters, not belonging to both limiting cases.

Although this phenomenon was the subject of many experimental and theoretical works, most of these concentrated on the two limiting cases. Especially there are only a few works treating this phenomenon exactly over wide ranges of the parameters.

Existing exact theories on the diffraction of light by ultrasound are along two lines: normal mode theory initiated by Brillouin ${ }^{5)}$ and plane wave theory based on the difference-differential equations of Raman and Nath. $\left.{ }^{6}, 7\right)$ Both theories reduce to Mathieu type differential equation and investigations were performed by Extermann and Wannier, ${ }^{8)}$ Extermann, ${ }^{9)}$ Wagner, ${ }^{10)}$ Plancke-Schuyten 
and Mertens, ${ }^{11)}$ Tamir and Wang, ${ }^{12)}$ Chu and Tamir $^{13)}$ and Nomoto. ${ }^{14-16)}$ Because of the mathematical difficulties, however, most of the work concentrated on the behaviour of the 1st and the 2nd-order diffraction spectrum, except for Nomoto $^{14-16)}$ and the semi-qualitative work of Wagner. ${ }^{10,10 a)}$

The purpose of the present paper is to present the result of a detailed calculation performed over a wide range of the parameters under normal incidence and under angles of incidences of various Bragg orders, to elucidate the nature of the Bragg maxima and minima, especially those of the higher order Bragg maxima and minima.

The experimental parameters pertaining to this phenomenon are the frequency $(f)$ and wavelength $(A)$ of sound, frequency $(\nu)$ and wavelength $(\lambda$, in the medium) of light, width $(L)$ of the sound beam in the plane of incidence of light, refractive index $\left(n_{0}\right)$ of the unperturbed medium and the amplitude of variation of it $(\mu)$ as determined by the pressure amplitude and the piezooptic constants-or the stress amplitude and the elastooptic constants in case of solids-, and the angle of incidence $(\phi$, in the medium) of light on the sound grating. The phenomenon depends also on whether the sound field is a progressive or a standing one. In what follows, however, we treat the more simple and fundamental case of the progressive sound wave exclusively. In other respects we consider here the fundamental two-dimensional model case as stated above.

The parameters $\lambda$ and $\Lambda$ determine the diffraction angle $\phi_{n}$ as dependent on the angle of incidence by

$$
\sin \left(\phi_{n}+\phi\right)=\sin \phi+n \frac{\lambda}{\Lambda}, \quad(n=0, \pm 1, \pm 2, \ldots)
$$

Here and in what follows all the angles are measured in the medium of sound.

The intensity distribution of the diffraction spectra amongst orders are determined, besides $\phi$, by the three ratios:

$$
\alpha=\frac{\lambda}{\Lambda}, \beta=\frac{\Lambda}{L} \text {, and } \gamma=\frac{\mu}{n_{0}},
$$

though the first parameter $\alpha$ has only slight influence on the intensity distribution as compared to the other two. Practically, however, some combinations of these three parameters:

$$
\begin{aligned}
& v=\frac{2 \pi \gamma}{\alpha \beta}=\frac{2 \pi \mu L}{n_{0} \lambda}, \\
& \theta=\frac{\gamma}{\alpha^{2}}=\frac{\mu \Lambda^{2}}{n_{0} \lambda^{2}}, \\
& \xi=\frac{2 \pi}{\beta} \sqrt{\gamma}=\frac{2 \pi}{\Lambda}\left(\frac{\mu}{n_{0}}\right)^{1 / 2} L,
\end{aligned}
$$

and

$$
D=\frac{\pi \alpha}{\beta}=\frac{\pi L \lambda}{\Lambda^{2}},
$$

or some other quantities nearly proportional or inversely proportional to these are of common use for the purpose of theoretical calculations. Of these four parameters, $v$ is the phase-lattice parameter in the Raman-Nath theory, ${ }^{4)}$ and $\xi$ is the geometrical optical parameter used in the rayoptics theory (Lucas and Biquard, ${ }^{3)}$ Nomoto, ${ }^{17)}$ Pouliquen and Segard ${ }^{18)}$ ). In the normal mode theory, on the other hand, $\theta$ and $D$ are the primary variables, because $\theta$ is related to the coefficient of the Mathieu equation describing the light propagation in the perturbed medium and $D$ is proportional to the phase changes between normal modes. So we have to use these two in the calculation together with $\alpha$. For the purpose of exhibiting the result, however, we adopted $v$ in place of $D$, and presented the result as equal intensity curves on the $\theta v$ diagram. On this diagram, $D=$ const represents straight line emerging from the origin and with gradient $2 D$ because of the relation

$$
v=2 D \theta,
$$

and $\xi=$ constant represents a parabola with abscissa as the axis because of

$$
\xi=\frac{v}{\sqrt{\theta}} .
$$

We see that only two out of the four variables $v$, $\theta, \xi$, and $D$ are independent.

\section{ANALYTICAL RESULT OF THE NORMAL MODE THEORY}

Neglecting the effect of propagation of sound, we write the refractive index in the medium occupied by an ultrasound beam of width $L$ propagating in the $z$-direction as

$$
n=n_{0}-\mu \cos K z \text {. }
$$




\section{O. NOMOTO and A. OUCHI: DIFFRACTION OF LIGHT BY ULTRASOUND}

The light wave in the sound field satisfies the scalar wave equation:

$$
\left.\begin{array}{l}
\frac{n^{2}}{c^{2}} \frac{\partial^{2} E}{\partial t^{2}}=\frac{\partial^{2} E}{\partial x^{2}}+\frac{\partial^{2} E}{\partial z^{2}}, \\
E_{z} \frac{\partial n^{2}}{\partial z}+n^{2} \operatorname{div} E=0
\end{array}\right\}
$$

in the approximation, that $(\partial / \partial z)\left[\left(1 / n_{0}\right)(\partial n / \partial z) E_{z}\right]$, etc. are small as compared to $\Delta E_{z}$ etc.

Resolving the light-amplitude in the sound field into harmonic components

$$
\begin{aligned}
E & =\sum_{\nu} c_{\nu} G\left(p_{\nu}, z\right) \exp i \omega\left[t-\left(p_{\nu} x+n_{0} \sin \phi \cdot z\right) / c\right] \\
& =\sum_{\nu} c_{\nu} G\left(p_{\nu}, z\right) \exp i \omega\left(t-p_{\nu} x / c\right) \cdot \exp (-i g K z)
\end{aligned}
$$

where

$$
\sin \phi=g \lambda / n_{0} \Lambda=2 g \sin \phi_{1}{ }^{B}
$$

we see that $G\left(p_{\nu}, z\right)=\exp i g K z \cdot f\left(p_{\nu}, z\right)$, so that (2.3) becomes

$$
E=\sum_{\nu} c_{\nu} f\left(p_{\nu}, z\right) \exp i \omega\left(t-p_{\nu} x / c\right) .
$$

This indicates that the light wave in the perturbed medium propagates in the $x$-direction, or parallel to the sound-wavefront. $f\left(p_{\nu}, z\right)$ satisfies the equation:

$$
\frac{d^{2} f}{d z^{2}}+h^{2}\left(n_{0}{ }^{2}-2 n_{0} \mu \cos K z-p_{\nu}{ }^{2}\right) f=0,
$$

which reduces to the standard-form Mathieu equation

$$
\frac{d^{2} f}{d \zeta^{2}}+(a-2 q \cos 2 \zeta) f=0
$$

by the substitutions:

$$
\begin{aligned}
& K z=\frac{2 \pi}{\Lambda} z=2 \zeta, \quad p_{\nu}{ }^{2}=n_{0}{ }^{2}-\frac{a_{\nu} \Lambda^{2}}{4 \lambda^{2}}, \\
& \frac{\mu \Lambda^{2}}{n_{0} \lambda^{2}}=\theta=\frac{q}{4} .
\end{aligned}
$$

The Mathieu equation (2.5) has stable solution only for certain ranges of the characteristic value $a=a_{\nu}$ of the constant $a$ and the solution depends on this characteristic value. Brillouin's theory-the normal mode theory-is systematically extended by Nomoto ${ }^{15}{ }^{16)}$ and the result is summarized in Table 1. The table here reproduced, however, is improved in two respects. Firstly, the mathematical normalization condition for the Mathieu function is separated from the physical boundary condition; and secondly, complete suffixes are employed so as to exclude any ambiguity.

The significant feature of the normal mode theory is that the light waves within the ultrasound-periodic medium - propagates always in the direction perpendicular to the sound propagation irrespective of the angle of incidence, i.e., the propagation direction outside the ultrasound. Each normal mode within the sound wave propagates with a characteristic velocity corresponding to the characteristic refractive index $p_{\nu}$ as determined by the characteristic value $a_{\nu}$ of the Mathieu function by (2.6).

The boundary condition at the plane of entrance, i.e., at the former boundary of the ultrasound beam, determines the nature of the Mathieu functions to be used. Even order Mathieu functions with characteristic values $a_{2 n^{\prime}}$,

$$
\mathrm{ce}_{2 n}(\zeta, q)=\sum_{r=0}^{\infty} A_{2 r}^{(2 n)}(q) \cos 2 r \zeta, \quad(n=0,1,2, \ldots)
$$

satisfy the boundary condition in case of the normal incidence. In case of the Bragg incidence of the even orders, functions $\mathrm{ce}_{2 n}$ and $\mathrm{se}_{2 n}$ cooperate in satisfying the boundary condition. Here $\mathrm{se}_{2 n}$ have characteristic values $b_{2 n}$ and have expressions:

$$
\operatorname{se}_{2 n}(\zeta, q)=\sum_{r=1}^{\infty} B_{2 r}^{(2 n)}(q) \sin 2 r \zeta, \quad(n=1,2, \ldots) .
$$

In case of the Bragg incidence of odd orders, functions with characteristic values $a_{2 n+1}$ and $b_{2 n+1}$, respectively, i.e.,

$$
\left.\begin{array}{c}
\operatorname{ce}_{2 n+1}(\zeta, q)=\sum_{r=0}^{\infty} A_{2 r+1}^{(2 n+1)}(q) \cos (2 r+1) \zeta, \\
(n=0,1,2, \ldots)
\end{array}\right\}
$$

and

$$
\left.\begin{array}{c}
\operatorname{se}_{2 n+1}(\cong, q)=\sum_{r=0}^{\infty} B_{2 r+1}^{(2 n+1)}(q) \sin (2 r+1) \zeta, \\
(n=0,1,2, \ldots)
\end{array}\right\}
$$

combined in the form $k_{2 n+1}^{(2 m+1)} \mathrm{ce}_{2 n+1}-i k_{2 n+1}^{\prime(2 m+1)} \mathrm{se}_{2 n+1}$ satisfy the boundary condition. Non-periodic functions $\mathrm{ce}_{2 n+\beta}$ and $\mathrm{se}_{2 n+\beta}$, or $\mathrm{ce}_{2 n+1+\beta}$ and $\mathrm{se}_{2 n_{+1+\beta}}$ appear under oblique incidences not corresponding to Bragg incidences. The notations here adopted 
Table 1 Normal mode Mathieu functions, boundary conditions, and the complex amplitudes of the diffraction spectra for various angles of incidence.

\begin{tabular}{|c|c|c|c|c|c|}
\hline \multirow{2}{*}{ Incidence } & \multirow{2}{*}{$\begin{array}{l}\text { Normal } \\
\text { incidence }\end{array}$} & \multicolumn{4}{|c|}{ Bragg incidence } \\
\hline & & \multicolumn{2}{|c|}{ Even orders } & \multicolumn{2}{|c|}{ Odd orders } \\
\hline $2 g$ & 0 & \multicolumn{2}{|c|}{$2 m(m=1,2,3, \ldots)$} & \multicolumn{2}{|c|}{$2 m+1(m=0,1,2,3, \ldots)$} \\
\hline $\begin{array}{l}\text { Characterist. } \\
\text { values }\end{array}$ & $a_{2 n}(q)$ & $a_{2 n}(q)$ & $b_{2 n}(q)$ & $a_{2 n+1}(q)$ & $b_{2 n+1}(q)$ \\
\hline \multirow[t]{2}{*}{$\begin{array}{l}\text { Normal mode } \\
\text { Mathieu } \\
\text { functions }\end{array}$} & $\begin{array}{l}k_{2 n}^{0} \mathrm{ce}_{2 n} \\
(n=0,1,2, \ldots)\end{array}$ & $\begin{array}{l}k_{2 n}^{2 m} \mathrm{ce}_{2 n} \\
(n=0,1,2, \ldots)\end{array}$ & $\begin{array}{l}-i k_{2 n}^{\prime 2 m} \mathrm{se}_{2 n} \\
(n=1,2, \ldots)\end{array}$ & $\begin{array}{l}k_{2 n+1}^{2 m+1} \mathrm{ce}_{2 n+1} \\
(n=0,1,2, \ldots)\end{array}$ & $\begin{array}{l}-i k_{2 n+1}^{\prime 2 m+1} \mathrm{se}_{2 n+1} \\
(n=0,1,2, \ldots)\end{array}$ \\
\hline & $\begin{array}{l}\mathrm{ce}_{2 n}(\zeta \\
=\sum_{r=0}^{\infty} A_{2}^{\varsigma}\end{array}$ & $\cos 2 r \zeta$ & $\begin{array}{l}\operatorname{se}_{2 n}(\zeta, q) \\
=\sum_{r=1}^{\infty} B_{2 r}^{(2 n)} \sin 2 r \zeta\end{array}$ & $\begin{array}{l}\operatorname{ce}_{2 n+1}(\zeta, q) \\
=\sum_{r=0}^{\infty} A_{2 r+1}^{(2 n+1)} \\
\cdot \cos (2 r+1) \zeta\end{array}$ & $\begin{array}{l}\operatorname{se}_{2 n+1}(\zeta, q) \\
=\sum_{r=0}^{\infty} B_{2 r+1}^{(2 n+1)} \\
\cdot \sin (2 r+1) \zeta\end{array}$ \\
\hline $\begin{array}{l}\text { Boundary } \\
\text { conditions }\end{array}$ & $k_{2 n}^{0}=2 A_{0}^{(2 n)}$ & $k_{2 n}^{2 m}=A_{2 m}^{(2 n)}$ & $k_{2 n}^{\prime 2 m}=B_{2 m}^{(2 n)}$ & $k_{2 n+1}^{2 m+1}=A_{2 m+1}^{(2 n+1)}$ & $k_{2 n+1}^{2 m+1}=B_{2 m+1}^{(2 n+1)}$ \\
\hline $\begin{array}{l}\text { Complex } \\
\text { amplitudes } \\
\text { of the } \\
\text { diffraction } \\
\text { spectrum }\end{array}$ & 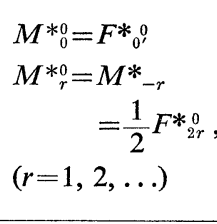 & $\begin{array}{r}M_{r-m}^{* 2 m}=\frac{1}{2}(F \\
M^{*-2 m}=F^{* 2 m} \\
M^{*-2 m}=\frac{1}{2} \\
\quad(r=1,2, .\end{array}$ & $\begin{array}{l}\left.{ }_{2 r}^{2 m}+G_{2 r}^{* 2 m}\right), \\
\left.F_{2 r}^{* 2 m}-G_{2 r}^{* 2 m}\right), \\
.)\end{array}$ & $\begin{array}{r}M_{r-m}^{* 2 m+1}=\frac{1}{2} \\
M_{-(r+m+1)}^{2 m+1}= \\
\quad(r=0,1,\end{array}$ & $\begin{array}{l}\left.F_{2 r+1}^{*_{2 m+1}^{2 m}}+G^{* 2 r+1}{ }_{2 r+1}^{2 m}\right), \\
\frac{1}{2}\left(F_{2 r+1}^{* 2 m+1}-G_{2 r+1}^{* 2 m+1}\right), \\
2, \ldots)\end{array}$ \\
\hline (where) & $\begin{aligned} F_{2 r}^{* 0} & = \\
& = \\
F^{*(2 m)} & = \\
& = \\
G_{n}^{*(2 m)} & = \\
& =\end{aligned}$ & $\begin{array}{l}F_{2 r}^{(2 n)(0)} \exp i\left(2 \phi_{2}\right. \\
k_{2 n}^{(0)} A_{2 r}^{(2 n)} \exp i(2 \varnothing \\
F_{2 r}^{(2 n), 2 m)} \exp i(2 \varphi \\
k_{2 n}^{(2 n n} A_{2 r}^{(2 n)} \exp i( \\
(r=0,1,2, \ldots) \\
G_{2 r}^{(2 n)(2 m)} \exp i(2\} \\
k_{2 n}^{(2 m)} B_{2 r}^{(2 n)} \exp i( \\
(r=1,2, \ldots)\end{array}$ & $\begin{array}{l}n), \\
\text { n) } \\
\left.\phi_{2 n}\right), \\
\psi_{2 n)},\end{array}$ & 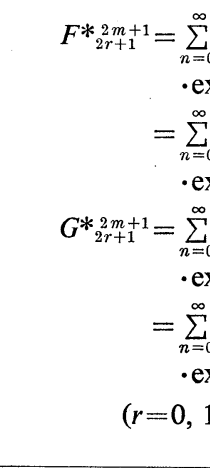 & $\begin{array}{l}F_{2 r+1}^{(2 m+1)(2 n+1)} \\
i\left(2 \phi_{2 n+1}\right) \\
k_{2 n+1}^{(2 m+1)} A_{2 r+1}^{(2 n+1)} \\
i\left(2 \phi_{2 n+1}\right) \\
G_{2 r+1}^{(2 m+1)(2 n+1)} \\
i\left(2 \psi_{2 n+1}^{(2 m)}\right. \\
k_{2 n+1}^{(2 m+1)} B_{2 r+1}^{(2 n+1)} \\
i\left(2 \psi_{2 n+1}^{(2 n)}\right. \\
2, \ldots)\end{array}$ \\
\hline (where) & $\begin{aligned} 2 \phi_{2 n} & =- \\
& =- \\
2 \psi_{2 n} & =-\end{aligned}$ & $\begin{array}{l}\frac{D}{2}\left[1-\left(1-\frac{1}{4} a_{2 n}\right.\right. \\
D\left(a_{2 n}+\frac{1}{16} \alpha^{2} a_{2 n}^{2}\right. \\
D\left(b_{2 n}+\frac{1}{16} \alpha^{2} b_{2 n}^{2}\right.\end{array}$ & $\begin{array}{l}\left.{ }^{1 / 2}\right] \\
\ldots), \\
\ldots)\end{array}$ & $\begin{array}{l}2 \phi_{2 n+1}=\frac{1}{4} L \\
2 \psi_{2 n+1}=\frac{1}{4} L\end{array}$ & $\begin{array}{l}\left(a_{2 n+1}+\frac{1}{16} \alpha^{2}\right. \\
\left.a_{2 n+1}^{2}+\ldots\right), \\
\left(b_{2 n+1}+\frac{1}{16} \alpha^{2}\right. \\
\left.b_{2 n+1}^{2}+\ldots\right)\end{array}$ \\
\hline
\end{tabular}

are the same with that of McLachlan ${ }^{19)}$ except for the enumeration of terms in (8). We deviated from McLachlan here for the purpose of maintaining the relation of the term number $2 r$ and the order of the spectrum common for $\mathrm{ce}_{2 n}$ and $\mathrm{se}_{2 n}$. Further we postulate the coefficients to satisfy the GoldsteinInce normalization conditions: 


\section{O. NOMOTO and A. OUCHI: DIFFRACTION OF LIGHT BY ULTRASOUND}

$$
\left.\begin{array}{l}
\sum_{2 n}=2\left(A_{0}^{(2 n)}\right)^{2}+\sum_{r=1}^{\infty}\left(A_{2 r}^{(2 n)}\right)^{2}=1, \\
(n=0,1,2, \ldots), \\
\sum_{2 n}^{\prime}=\sum_{r=1}^{\infty}\left(B_{2 r}^{(2 n)}\right)^{2}=1, \quad(n=1,2, \ldots), \\
\sum_{2 n+1}=\sum_{r=0}^{\infty}\left(A_{2 r+1}^{(2 n+1)}\right)^{2}=1, \quad(n=0,1,2, \ldots), \\
\sum_{2 n+1}^{\prime}=\sum_{r=0}^{\infty}\left(B_{2 r+1}^{(2 n+1)}\right)^{2}=1, \quad(n=0,1,2, \ldots) .
\end{array}\right\}
$$

The proportionality constants are determined from the boundary condition at the plane of entrance into ultrasound, together with the kind of the Mathieu functions to be employed. For example, in case of the 2nd-order Bragg incidence,

$$
\begin{aligned}
& {\left[\sum_{n=0}^{\infty} k_{2 n} \mathrm{ce}_{2 n}(\zeta, q) \exp \left(-i \omega p_{2 n} x / c\right)\right.} \\
& \left.\quad-i \sum_{n=1}^{\infty} k_{2 n}^{\prime} \operatorname{se}_{2 n}(\zeta, q) \exp \left(-i \omega p_{2 n}^{\prime} x / c\right)\right]_{x=0} \\
& \quad=\exp (-i 2 \zeta)
\end{aligned}
$$

In crossing the ultrasound beam, the normal modes of light waves produce phase differences among them. In Table $1,2 \phi_{2 n}, 2 \psi_{2 n^{\prime}}$, etc. indicate the phase differences with respect to the plane wave in unperturbed medium. We see that the phase differences are proportional to the parameter $D$. The influence of the parameter $\alpha$ is through its small influence on the phase difference, though at very high frequencies, when $\alpha$ becomes large, the phase difference and therefore also the intensity distribution changes appreciably. Strictly speaking, we can construct the $\theta v$-diagram only for predetermined experimental conditions, and varying the value of $\alpha$ at each point of the diagram. This, however, will limit the universality of the result appreciably. Fortunately, however, the phasedifference and the intensity distribution is nearly independent of the choice of the parameter $\alpha$, and it is more convenient to use a single value of $\alpha$ throughout the diagram. We adopted $\alpha=0.003$ in the calculation. This corresponds to $10 \mathrm{MHz}$ in a medium with sound velocity $1500 \mathrm{~m} / \mathrm{s}$ and $n_{0}=$ 1.333 when light source of vacuum wavelength 6000 $\AA$ is employed. The result, however, is equally valid for $100 \mathrm{MHz}$ and for $1 \mathrm{MHz}$, too. In the present calculation, we adopted normal modes $\mathrm{ce}_{0}, \mathrm{ce}_{2}, \ldots$, $\mathrm{ce}_{14}$ and $\mathrm{se}_{2}, \mathrm{se}_{4}, \ldots, \mathrm{se}_{14}$ in case of the even order
Bragg incidence, and $\mathrm{ce}_{1}, \mathrm{ce}_{3}, \ldots, \mathrm{ce}_{15}$ and $\mathrm{se}_{1}, \mathrm{se}_{3}, \ldots$, $\mathrm{Se}_{15}$ in case of the odd order Bragg incidence, in the range of the parameter $0<\theta \leqq 10$. All characteristic values $a_{\nu}, b_{\nu}$ and the expansion coefficients $A_{k}, B_{k}$ corresponding to these Mathieu functions were calculated as functions of $q(=4 \theta)$ by an improved method as devised by one of the authors (Ouchi). (cf. also Yamamoto ${ }^{8 a}$ for the method of obtaining the characteristic values.)

\section{DISTRIBUTION OF THE MAXIMUM- INTENSITY AREAS OF THE BRAGG-REFLECTED LIGHT}

\subsection{General Aspects of the Phenomenon}

It was first pointed out by Extermann ${ }^{9)}$ that the ultrasonic light diffraction spectrum not necessarily becomes maximum under Bragg incidence, but sometimes becomes minimum at the Bragg angle. Plancke-Schuyten and Mertens ${ }^{11)}$ also obtained similar results. Both calculations are performed for the case $\theta=1$, and Extermann's result is that the first order spectrum becomes maximum at Bragg incidence if the value of $D$ is $D=5.9$, but minimum if $D=15.2$, and that the 2nd-order spectrum under 2nd-order Bragg incidence becomes maximum if $D=9$, but minimum at $D=24.9$.

The present result shows that the areas of great intensity and those of almost vanishing intensity of the Bragg reflected light are distributed over the $\theta v$-plane and even a slight variation of the parameters are liable to cause great variation of the light intensity.

Figure 1 shows the areas of maximum intensity of the 1st, 2nd, 3rd and 4th-order spectrum under Bragg incidences of respective order spectra. Also the areas of maximum intensity of the 0 -order spectrum under normal incidence are indicated. Raman-Nath peaks and Raman-Nath-like peaks are located below the curve $\xi=2$ and are not indicated here. These are of minor intensity and located nearly parallel to the $\theta$-axis.

It is to be noticed that the areas of maximum intensity of the Bragg diffracted light are distributed nearly along the curves $\xi=$ const. Especially the 1 st non-Raman-Nath-type maximum corresponding to the lowest value of $v$ along the curve $\xi=4$ is apparent. Even more prominent maximum are discernible around the other curves $\xi=12$, and $\xi=20$. It must be added here that each order spectrum under Bragg incidence have large intensity values 


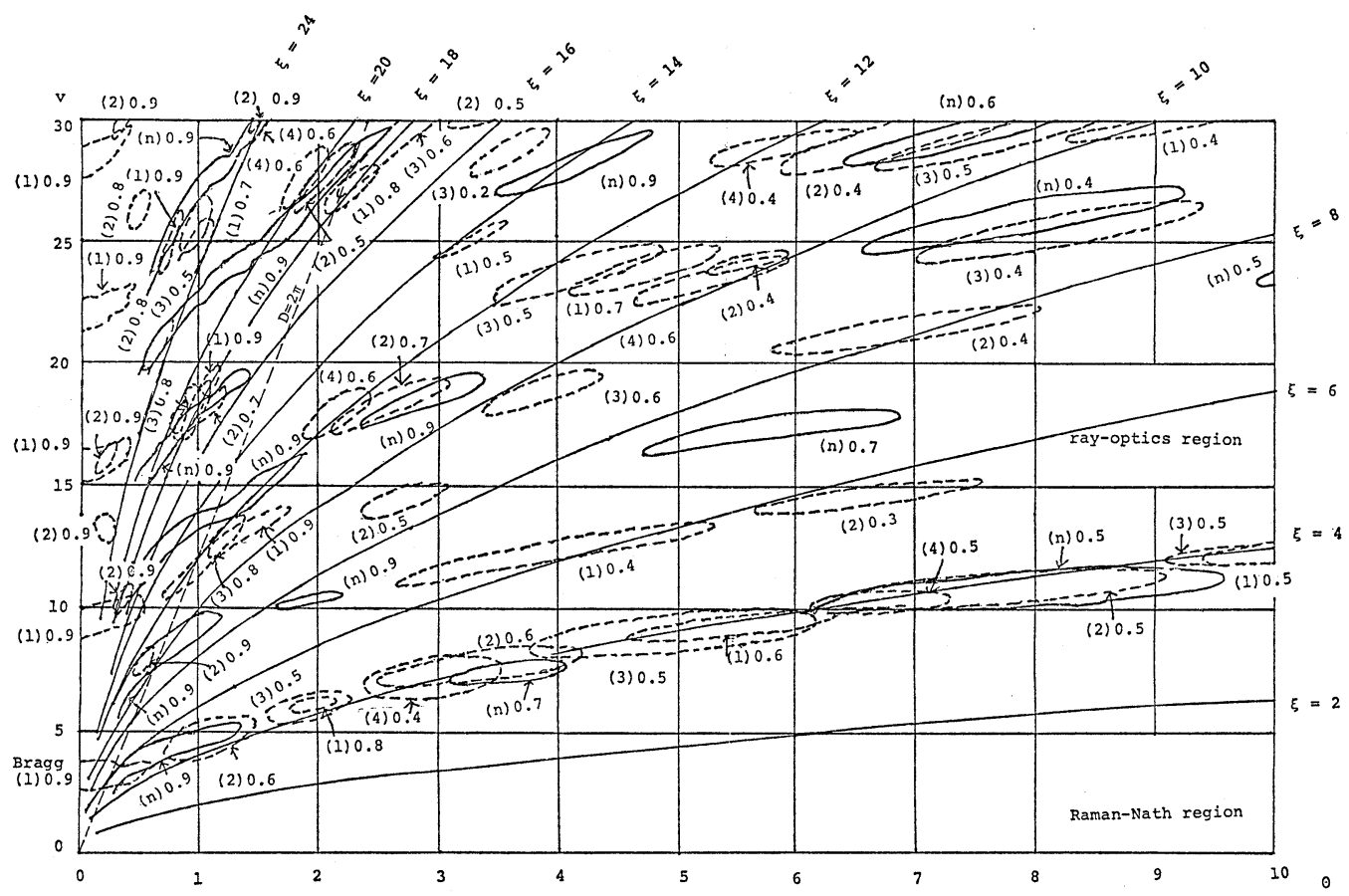

Fig. 1 Locations of the areas of Bragg maxima for the 1st, 2nd, 3rd and 4th-order spectra on the $\theta v$-diagram.

The numbers in the parentheses, $(1) \sim(4)$, indicate the Bragg orders and $(n)$ indicates the 0 -order under normal incidence. The figures outside the parentheses indicate the intensity along the enclosure. For example, (1) 0.9 indicates that the intensity of the 1st-order Bragg maximum is higher than 0.9 (90\% the incident light) within the enclosed area.

not only in the indicated areas, but also along the whole length of the curves $\xi=$ const, the indicated areas being, so to speak, the peaks along the mountain ranges. At least, the vanishing does not occur on these curves, but takes place along other curves with different values of $\xi$.

The 1st-order spectrum under 1st-order Bragg incidence exhibits maximum also in areas along the ordinate axis $(\theta=0)$ and located nearly at equal distances: $v=\pi, 3 \pi, 5 \pi$, etc. These are the highest maxima for the 1st-order spectrum attaining nearly $100 \%$ of the incident light, and may be termed the "true Bragg reflection", while the maxima located along the curves $\xi=$ const may rather be called the "mirror type Bragg maxima" as discussed later. Willard $^{20)}$ has pointed out the geometrical condition for the true Bragg reflection as $L \phi_{1}{ }^{B}=L \lambda$ / $2 \Lambda \geqq \Lambda$, or $D \geqq 2 \pi$ in our notation. This corresponds to the condition that at least two Bragg reflected rays do exist to reinforce among them. We see in
Fig. 1 that the maxima along the curves $\xi=$ const are discernible also in the region satisfying this condition. The main part of the maxima except for the 1st-order spectrum, however, are located outside the region satisfying the Willard's condition, and we infer that the mechanism of Bragg reflection in case of the light diffraction by ultrasound is rather different from that of the Bragg reflection in the geometrical meaning.

Figure 2 shows the equal-intensity curves on $\theta v$ diagrams for the 0 -order spectrum under normal incidence. Traces of the maxima-obtained along the line $\theta=$ const-and minima are also indicated by triangles and inverted triangles, respectively. These curves were obtained as computer plots. The equal-intensity curves consist of small segments with directions tangential to the curves. The thick curves on the other hand, were obtained by independent calculation and constructed by hand plotting afterwards. The thick curves represent the loci 


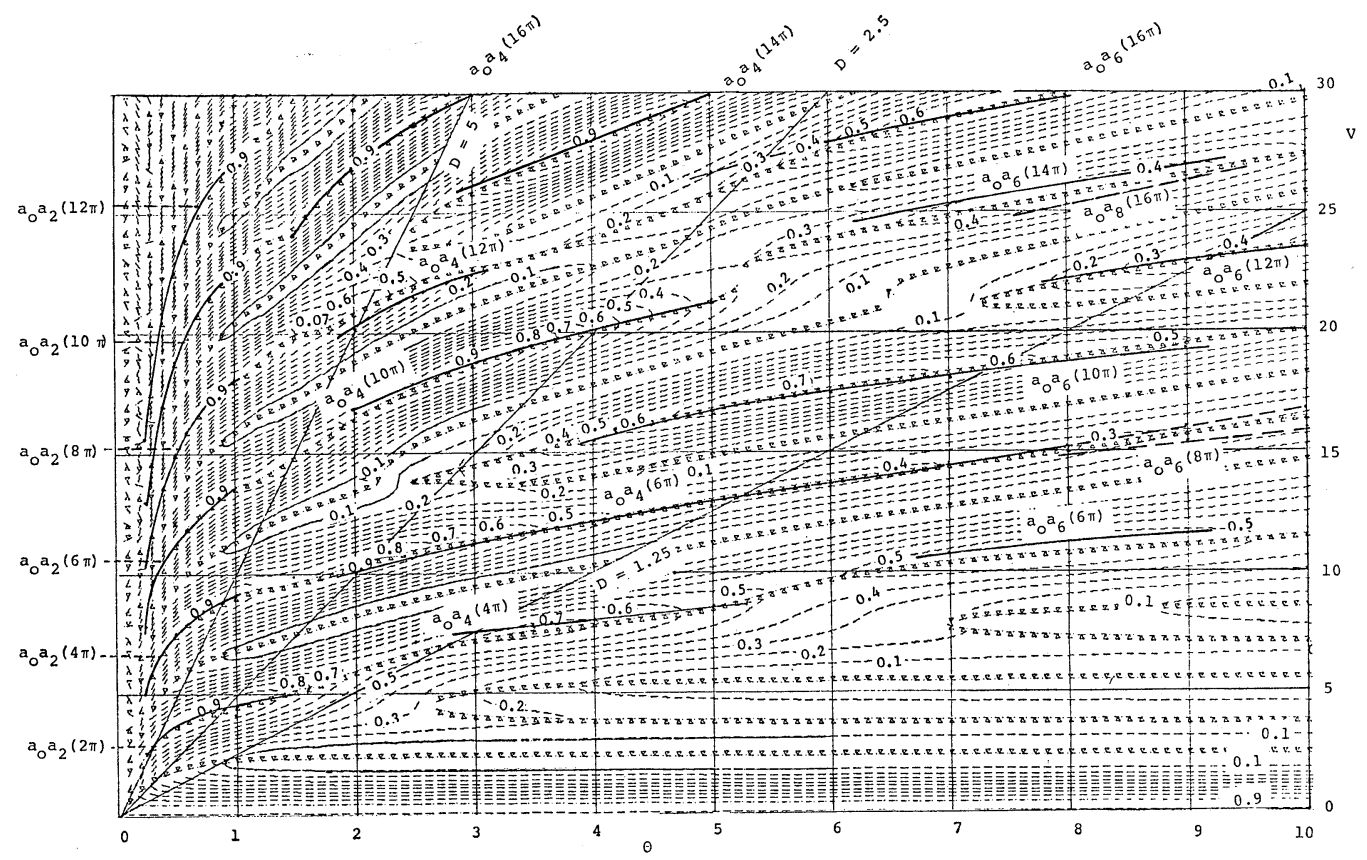

Fig. 2 Intensity distribution on the $\theta v$-diagram: Normal incidence, 0 -order spectrum.

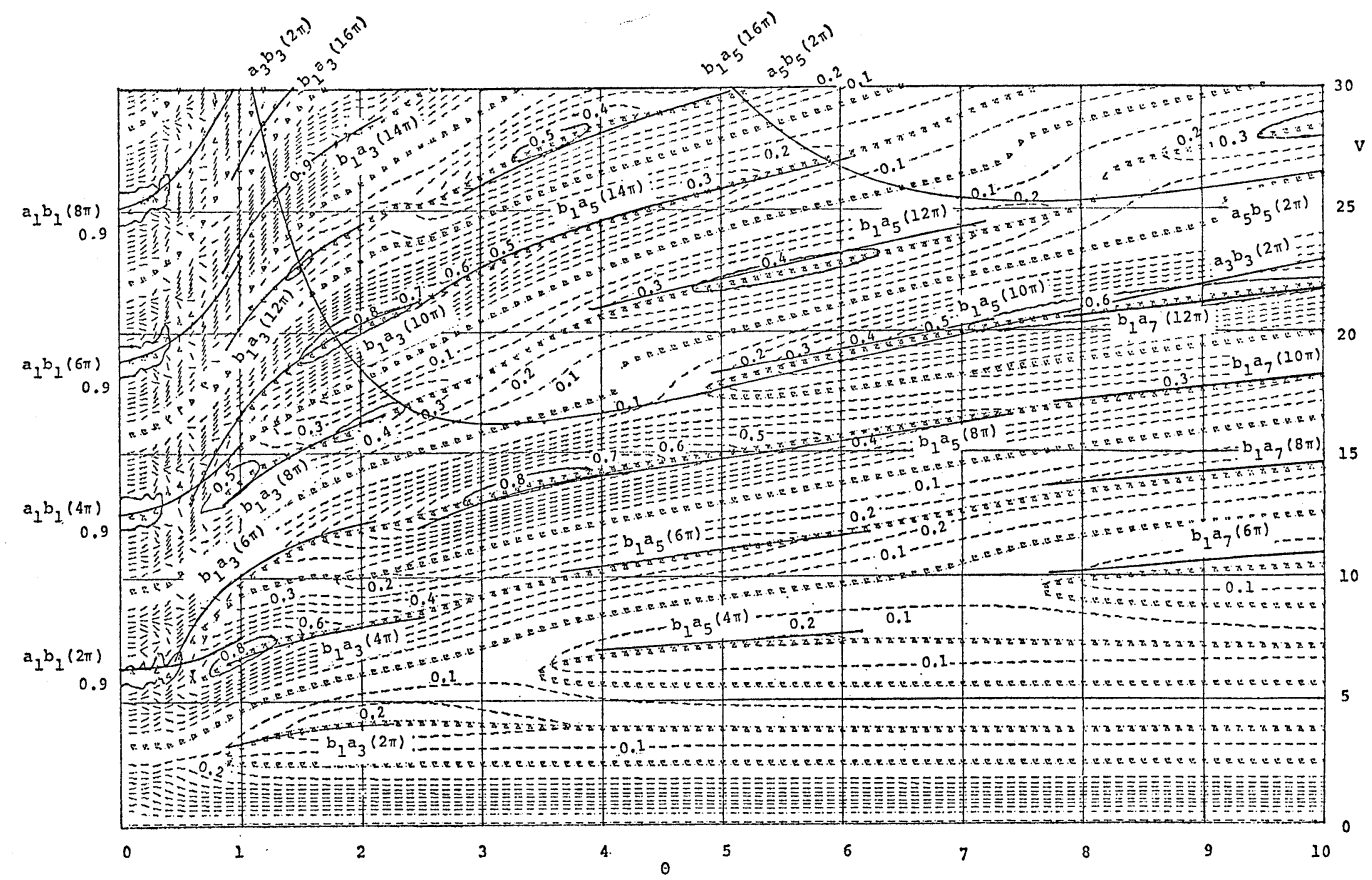

Fig. 3 Intensity distribution on the $\theta v$-diagram: 1st order Bragg incidence, 0-order spectrum. 


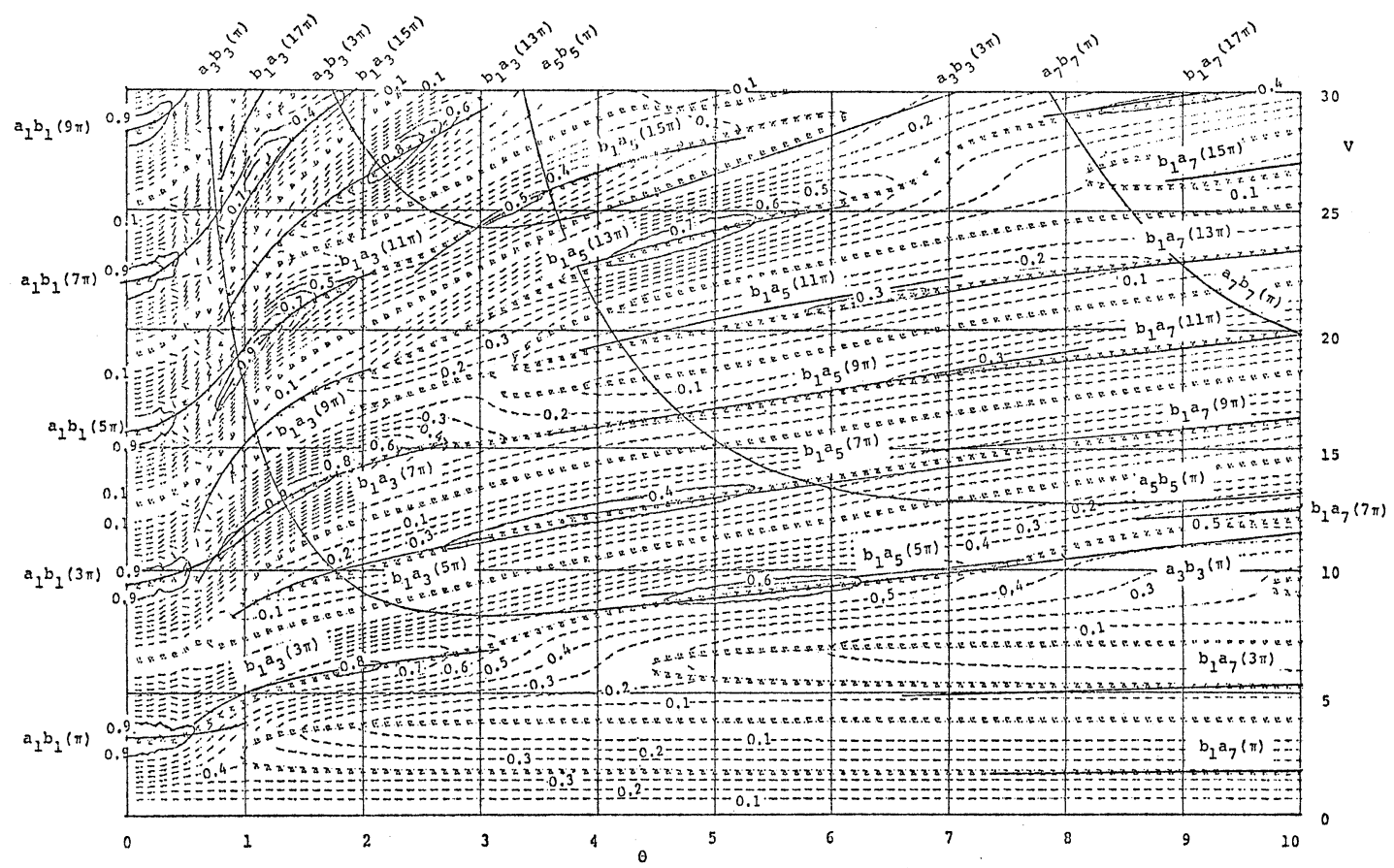

Fig. 4 Intensity distribution on the $\theta v$-diagram: 1st-order Bragg incidence, Bragg-side 1st-order spectrum.

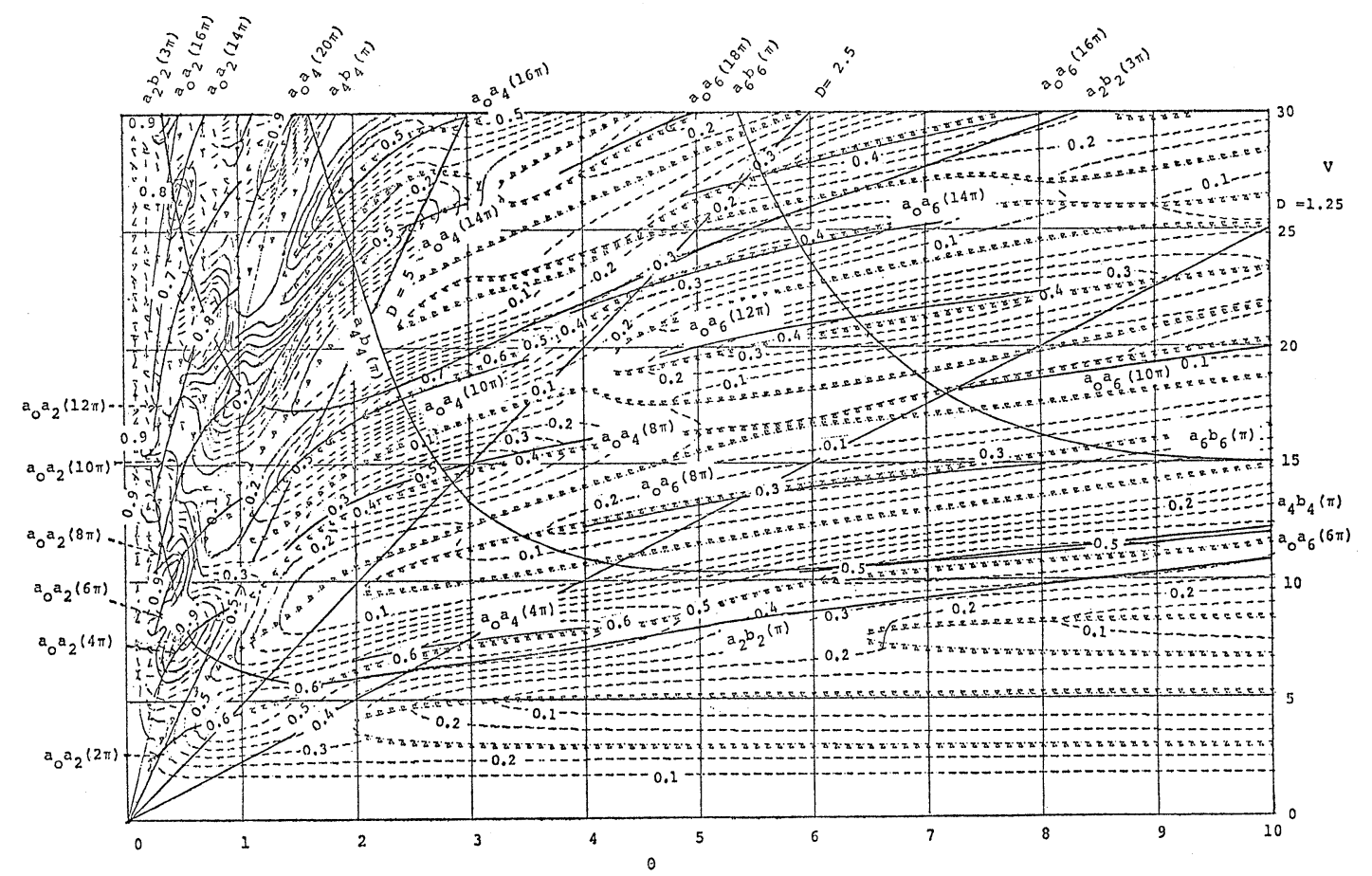

Fig. 5 Intensity distribution on the $\theta v$-diagram: 2nd-order Bragg incidence, Bragg-side 2nd-order spectrum. 


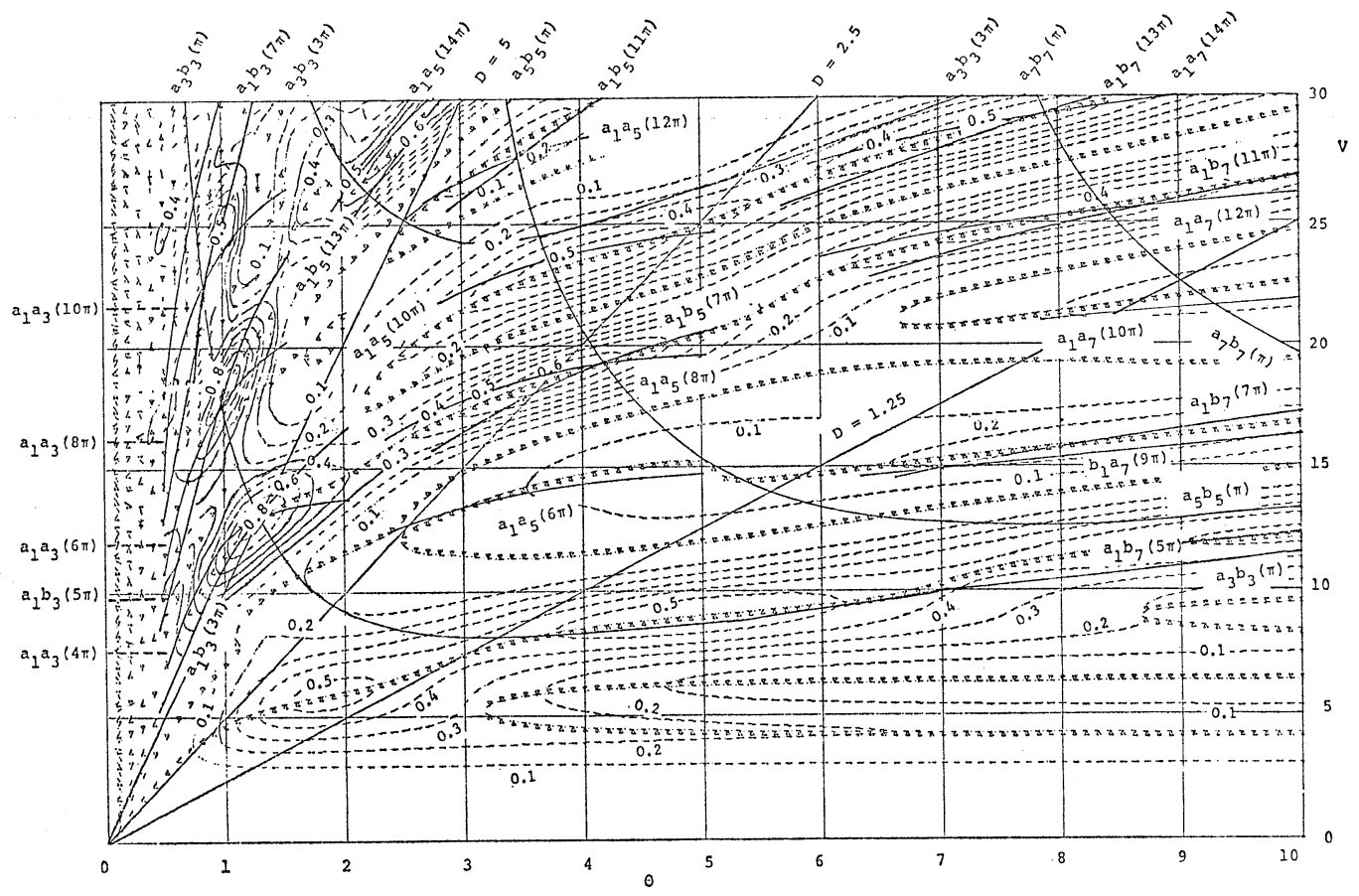

Fig. 6 Intensity distribution on the $\theta v$-diagram: 3rd-order Bragg incidence, Bragg-side 3rd-order spectrum.

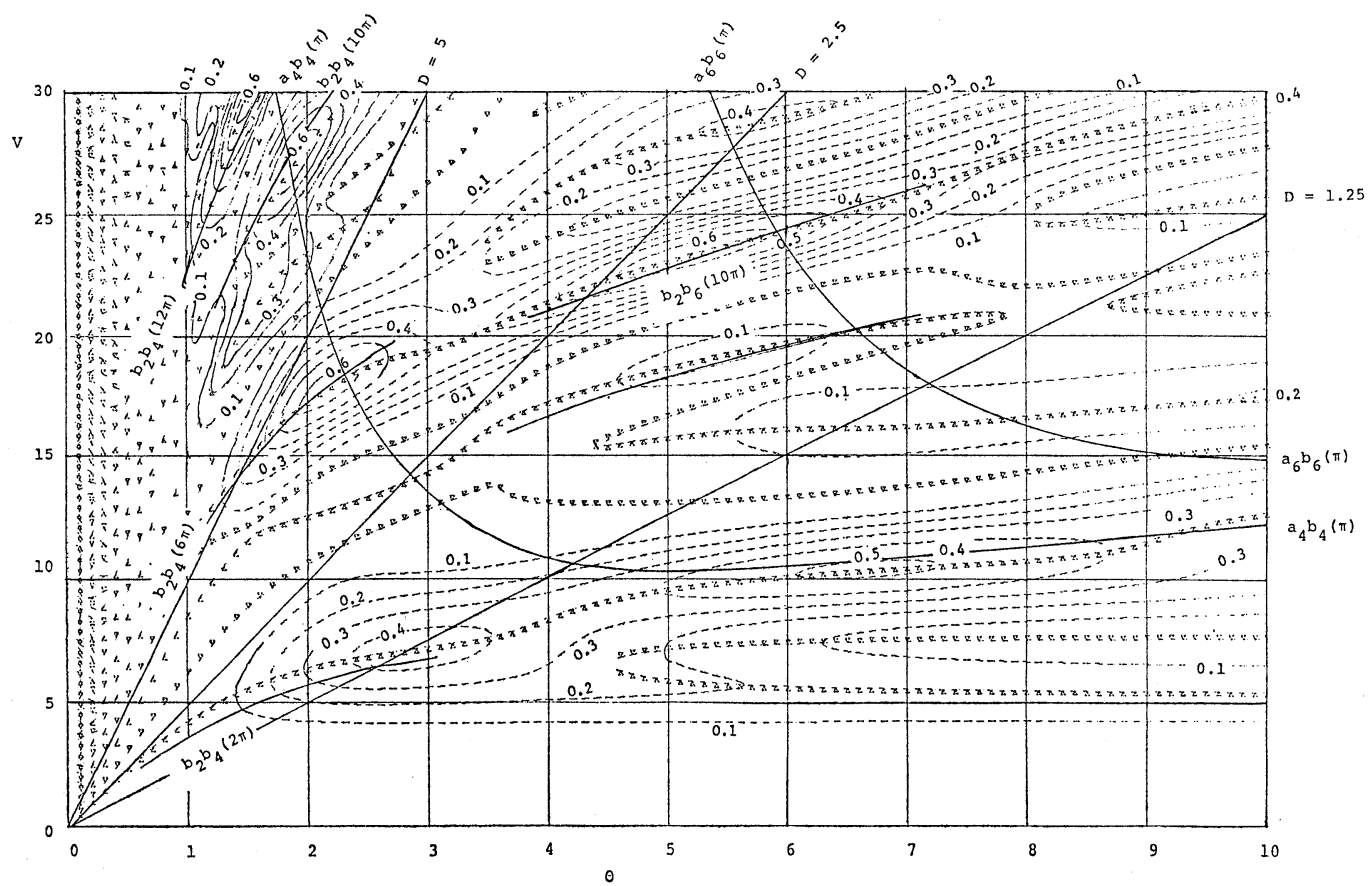

Fig. 7 Intensity distribution on the $\theta v$-diagram: 4th-order Bragg incidence, Bragg-side 4th-order spectrum. 
Table 2 Relation of the spectrum order with the term number $(2 r$ or $2 r+1)$ of the Mathieu functions under various angles of incidence $(2 m$ th or $(2 m+1)$ th order Bragg incidence).

\begin{tabular}{|c|c|c|c|c|c|c|c|c|c|}
\hline \multirow{3}{*}{\multicolumn{2}{|c|}{$\begin{array}{c}\text { Term } \\
\text { numbers } \\
\text { (cf. Eq. }(2.7),(2.8))\end{array}$}} & \multicolumn{6}{|c|}{ Bragg incidence orders } & \multirow{3}{*}{\multicolumn{2}{|c|}{$\begin{array}{c}\text { Term } \\
\text { numbers } \\
\text { (cf. Eq. }(2.9),(2.10))\end{array}$}} \\
\hline & & \multicolumn{3}{|c|}{ even orders } & \multicolumn{3}{|c|}{ odd orders } & & \\
\hline & & \multirow{2}{*}{$\begin{aligned} 2 m & =0 \\
m & =0\end{aligned}$} & \multirow{2}{*}{$\begin{array}{l}2 \\
1\end{array}$} & \multirow{2}{*}{$\begin{array}{l}4 \\
2\end{array}$} & \multirow{2}{*}{$\begin{array}{c}2 m+1=1 \\
m=0\end{array}$} & \multirow{2}{*}{$\frac{3}{1}$} & \multirow{2}{*}{$\begin{array}{l}5 \\
2\end{array}$} & & \\
\hline & & & & & & & & & \\
\hline $2 r=14$ & $r=7$ & 7 & 6 & 5 & 7 & 6 & 5 & $r=7$ & $2 r+1=15$ \\
\hline 12 & 6 & 6 & 5 & 4 & 6 & 5 & 4 & 6 & 13 \\
\hline 10 & 5 & 5 & 4 & 3 & 5 & 4 & 3 & 5 & 11 \\
\hline 8 & 4 & 4 & 3 & 2 & 4 & 3 & 2 & 4 & 9 \\
\hline 6 & 3 & 3 & 2 & 1 & 3 & 2 & 1 & 3 & 7 \\
\hline 4 & 2 & 2 & 1 & 0 & 2 & 1 & 0 & 2 & 5 \\
\hline 2 & 1 & 1 & 0 & -1 & 1 & 0 & -1 & 1 & 3 \\
\hline \multirow[t]{2}{*}{0} & 0 & 0 & -1 & -2 & 0 & -1 & -2 & 0 & 1 \\
\hline & & & & & -1 & -2 & -3 & 0 & 1 \\
\hline 2 & 1 & -1 & -2 & -3 & -2 & -3 & -4 & 1 & 3 \\
\hline 4 & 2 & -2 & -3 & -4 & -3 & -4 & -5 & 2 & 5 \\
\hline 6 & 3 & -3 & -4 & -5 & -4 & -5 & -6 & 3 & 7 \\
\hline 8 & 4 & -4 & -5 & -6 & -5 & -6 & -7 & 4 & 9 \\
\hline 10 & 5 & -5 & -6 & -7 & -6 & -7 & -8 & 5 & 11 \\
\hline 12 & 6 & -6 & -7 & -8 & -7 & -8 & -9 & 6 & 13 \\
\hline 14 & 7 & -7 & -8 & -9 & -8 & -9 & -10 & 7 & 15 \\
\hline
\end{tabular}

of the points where two of the normal modes interfere to reinforce each other, as will be discussed in 3.2 .

Figure 3 represents the intensity distribution over the $\theta v$-plane of the 0 -order spectrum in case of the Bragg incidence of the 1st-order. Figures $4 \sim 7$ show similar graphs for the 1st, 2nd, 3rd and 4th-order spectra under their respective Bragg incidence angles. Figure 1, reproduced before, has been constructed from these results.

\subsection{Interpretation of the Location of Maxima of the Light Intensity}

Each of the harmonic terms constituting the Fourier components of Mathieu functions contributes to the diffraction spectrum of a definite order, a certain order spectrum being composed of contributions from harmonics of the same periodicity coming from various normal modes. For example, the 0 -order and the -1 st $\left(1^{B}\right)$-order-the Bragg order in our notation-comes from the terms containing $\cos \zeta$ and $\sin \zeta$ of various normal modes. The relation between the term number and the spectrum order is indicated for various angles of incidences in Table 2. Corresponding to each term number $2 r$ or $2 r+1$, there are usually two spectra with orders $r-m$ and $-(r+m)$ or with orders $r-m$ and $-(r+m+1)$, respectively, under the same incidence angles $-2 m$ th or $(2 m+1)$ th-order Bragg incidence (cf. Table 1). These spectra are complementary in the meaning that the complex amplitudes are proportional to $F^{*}+G^{*}$ and $F^{*}-G^{*}$ respectively, where $F^{*}$ comes from the ce-functions and $\mathrm{G}^{*}$ from the se-functions.

As the complex amplitude of the diffraction spectrum is composed as the vector sum of many components coming from various normal modes, exact value of the complex amplitude is only obtainable by taking all terms into account. Practically, modes up to $\mathrm{ce}_{14}$ and $\mathrm{se}_{14}$ or $\mathrm{ce}_{15}$ and $\mathrm{se}_{15}$ were taken into account in the above computation. The approximate location of the maximum, however, is usually determined by a small number of terms, especially in the range of smaller values of $\theta$. If only a small number of terms contribute to the complex amplitudes of a certain order spectrum, then we may expect that the amplitude will take maximum value nearly under the condition that two out of 


\section{O. NOMOTO and A. OUCHI: DIFFRACTION OF LIGHT BY ULTRASOUND}

Table 3 Condition for the mutual reinforcement of the contributions from various normal modes for the Bragg order spectrum and for the 0 -order.

\begin{tabular}{|c|c|}
\hline Incidence & Bragg order spectrum \\
\hline Normal incidence & $\begin{array}{l}\frac{D}{4}\left(a_{2 n}-a_{2 n^{\prime}}\right)=0,2 \pi, 4 \pi, \ldots \\
\quad n, n^{\prime}=0,1,2, \ldots ;\left(n \neq n^{\prime}\right)\end{array}$ \\
\hline \multirow[t]{2}{*}{$\begin{array}{l}\text { Even order Bragg } \\
\text { incidence }\end{array}$} & $\begin{array}{l}\frac{D}{4}\left(a_{2 n}-b_{2 n^{\prime}}\right)=0,2 \pi, 4 \pi, \ldots \\
\quad n=0,1,2, \ldots ; n^{\prime}=1,2, \ldots\end{array}$ \\
\hline & $\begin{array}{l}\frac{D}{4}\left(a_{2 n}-a_{2 n^{\prime}}\right)=0,2 \pi, 4 \pi, \ldots ; n, n^{\prime}=0,1,2, \ldots,\left(n \neq n^{\prime}\right) \\
\frac{D}{4}\left(b_{2 n}-b_{2 n^{\prime}}\right)=0,2 \pi, 4 \pi, \ldots ; n, n^{\prime}=1,2,3, \ldots,\left(n \neq n^{\prime}\right)\end{array}$ \\
\hline \multirow{2}{*}{$\begin{array}{l}\text { Odd order Bragg } \\
\text { incidence } \\
\left(n, n^{\prime}=0,1,2, \ldots\right)\end{array}$} & $\frac{D}{4}\left(a_{2 n+1}-b_{2 n^{\prime}+1}\right)=\pi, 3 \pi, 5 \pi, \ldots \quad \quad \frac{D}{4}\left(a_{2 n+1}-b_{2 n^{\prime}+1}\right)=0,2 \pi, 4 \pi, \ldots$ \\
\hline & $\begin{array}{l}\frac{D}{4}\left(a_{2 n+1}-a_{2 n^{\prime}+1}\right)=0,2 \pi, 4 \pi, \ldots,\left(n \neq n^{\prime}\right) \\
\frac{D}{4}\left(b_{2 n+1}-b_{2 n^{\prime}+1}\right)=0,2 \pi, 4 \pi, \ldots,\left(n \neq n^{\prime}\right)\end{array}$ \\
\hline
\end{tabular}

these normal modes reinforce each other. Such conditions for mutual reinforcement depends also on the signs of the coefficients, $F_{2 r}^{(2 n)(2 m)}$ and $G_{2 r}^{(2 n)(2 m)}$, for example, in general cases. In case of the Bragg orders and the 0-order, however, the situation is more simple because all these individual coefficients are positive, because of the relations: $F_{2 r}^{(2 n)(2 m)}=F_{2 m}^{(2 n)(2 m)}=k_{2 n}^{(2 m)} A_{2 m}^{(2 n)}=\left[A_{2 m}^{(2 n)}\right]^{2}$, etc., since $r=m$ in these cases. So we have Table 3 as the condition for mutual reinforcement of the normal mode components for the Bragg order and the 0-order spectrum under even and odd order Bragg incidence. The thick curves in Figs. $2 \sim 7$ are constructed in this manner. The notations in the Figures are abridged. For example $a_{1} b_{1}(9 \pi)$ means that the curve corresponds to $(D / 4)\left(a_{1}-b_{1}\right)=9 \pi$. The notation also includes the information on the both normal modes contributing to this maximum. For example, $a_{1} a_{5}(6 \pi)$ (Fig. 6) means that the locus of this curve corresponds to the reinforcement of the components coming from the normal modes $\mathrm{ce}_{1}$ with characteristic value $a_{1}$ and the mode $\mathrm{ce}_{5}$ with characteristic value $a_{5}$. This result indicates that many of the thick curves thus constructed are in good accord with the traces of the maximum obtained by computer calculation employing all normal modes. The selection of the normal modes, how- ever, is not unique solution in some cases. They were selected from possible combinations satisfying theoretical demands of Table 3 to be in good agreement with the computer result, also considering of the magnitude of the terms.

In regions of small $\theta$, the group of curves $a_{n} b_{n}$ ( $\pi)$ or $a_{n} b_{n}(2 \pi)$ usually does not give direct maximum, except for the cases $a_{1} b_{1}(\pi)$ and $a_{1} b_{1}(2 \pi)$ to be discussed later.

These curves, however, are of use in elevating the maximum level of the intersecting curves, if the latter has one common normal mode with the former. For example, the intersection of $b_{1} a_{3}(7 \pi)$ with $a_{3} b_{3}(\pi)$ (Fig. 4) must elevate the maximum of the former because at the point of intersection three normal modes $\mathrm{se}_{1}\left(b_{1}\right), \mathrm{ce}_{3}\left(a_{3}\right)$ and $\mathrm{se}_{3}\left(b_{3}\right)$ reinforce altogether. There are, however, cases when some out of the same family of curves correspond to only very low peaks, while the others indicate very strong ones. For example, $b_{1} a_{3}(9 \pi)$ (Fig. 4) is very weak, while $b_{1} a_{3}(7 \pi)$ and $b_{1} a_{3}(11 \pi)$ are very strong. This indicates, that there are also other modes than $\mathrm{se}_{1}$ and $\mathrm{ce}_{3}$ (in this case also $\mathrm{se}_{3}$ because of the intersection with $\left.a_{3} b_{3}(\pi)\right)$ that contribute to the peak in this case. If other modes cooperate to diminish the maximum, weak maximum or even vanishing of the peak is expectable. 


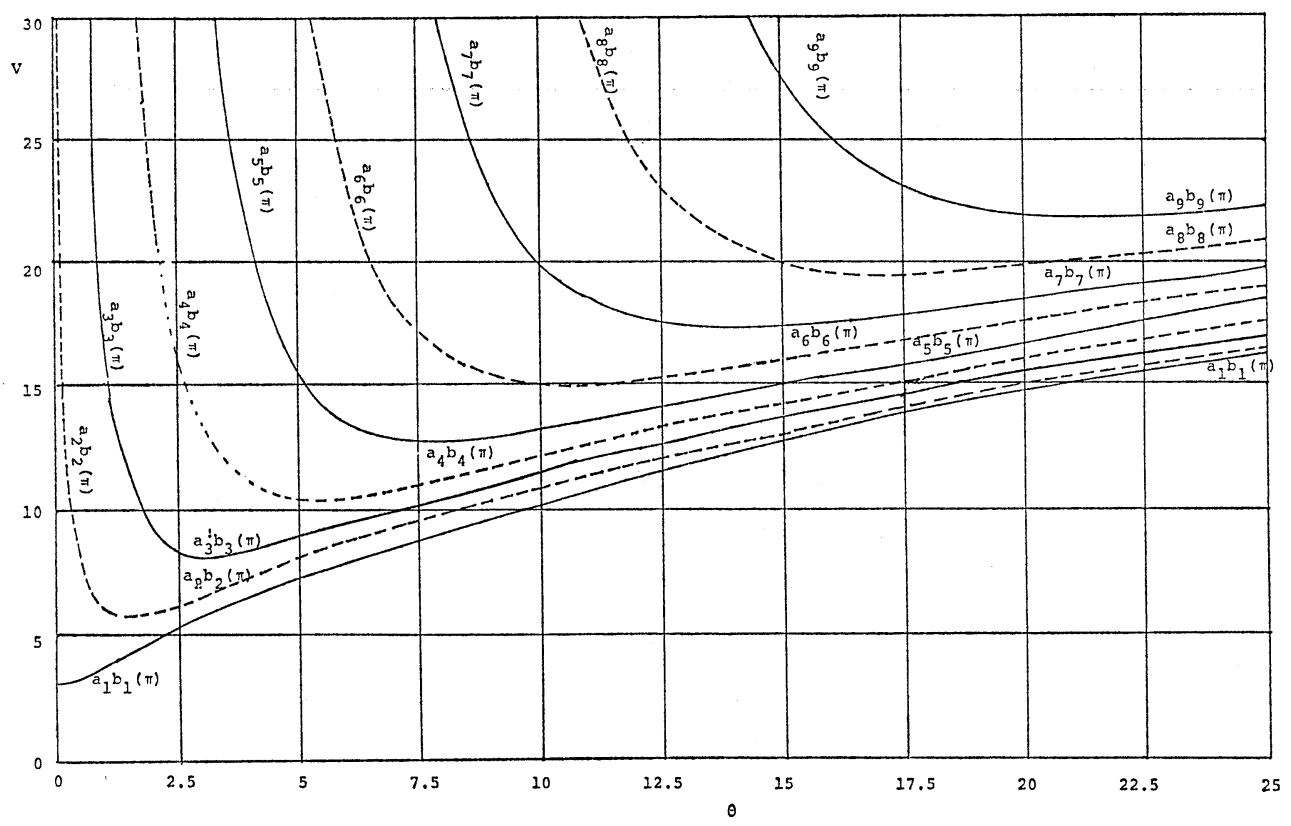

Fig. 8 Group of curves indicating the loci of points satisfying $(D / 4)\left(a_{n}-b_{n}\right)=\pi$, the condition for mutual reinforcement of the $n$-th harmonic components of the $\mathrm{ce}_{n}$ - and $\mathrm{se}_{n}$-normal modes.

Continuous curves are for the odd Bragg-order incidences and the dotted curves are for the even order Bragg incidences.

Figure 8 shows the group of curves $a_{n} b_{n}(\pi)$ on the $\theta v$-plane for a wider range of $\theta(\leqq 25)$. Characteristic values in this case were obtained from published tables. ${ }^{21}$ ) We see that these curves have two branches, except for the case of $a_{1} b_{1}(\pi)$. One branch approaches $v=\infty$, as $\theta$ becomes small, while the other branch form a nearly parallel bundle, with center of the bundle located in the neighbourhood of the curve $\xi=4$. This second branch is associated with the Bragg maximum for higher orders.

The circumstance in case of the 1st-order Bragg maximum is unique that the highest maxima of nearly $100 \%$ of the incident light is located at equal distances along the ordinate axis $(\theta=0)$ (cf. Fig. 4). This, however, is the result of the dependency of the characteristic values $a_{1}$ and $b_{1}$ on the value of $\theta$. The condition of reinforcement in this case being, $(D / 4)\left(a_{1}-b_{1}\right)=(2 n+1) \pi$ (cf. Table 2), we can transform it as

$$
\frac{v}{8 \theta}\left(a_{1}-b_{1}\right)=(2 n+1) \pi
$$

by means of Eq. (1.7). Now we have as the expansion of the characteristic values (cf. McLachlan ${ }^{19}$ ),

$$
\left.\begin{array}{l}
a_{1}=1+q-\frac{q^{2}}{8}-\frac{q^{3}}{64} \pm \ldots, \\
b_{1}=1-q-\frac{q^{2}}{8}+\frac{q^{3}}{64} \pm \ldots,
\end{array}\right\}
$$

so that we have

$$
a_{1}-b_{1}=2 q-\frac{q^{3}}{32} \pm \ldots .
$$

Considering that $q=4 \theta$ (cf. Eq. (2.6)), we have in place of (3.1),

$$
v=(2 n+1) \pi,
$$

as revealed in the Fig. 4. We do not encounter the same circumstance in other cases, because $a_{2}, b_{2}, \ldots$ all lack terms proportional to $q$ in the expansions.

The other normal modes being only small in the range $\theta \ll 1$, the maxima and minima in the neighbourhood of the ordinate axis is determined by $\mathrm{ce}_{1}$ and $\mathrm{se}_{1}$ almost exclusively. So the variation of the 1st-order (Bragg order) and the 0-order is 


\section{O. NOMOTO and A. OUCHI: DIFFRACTION OF LIGHT BY ULTRASOUND}

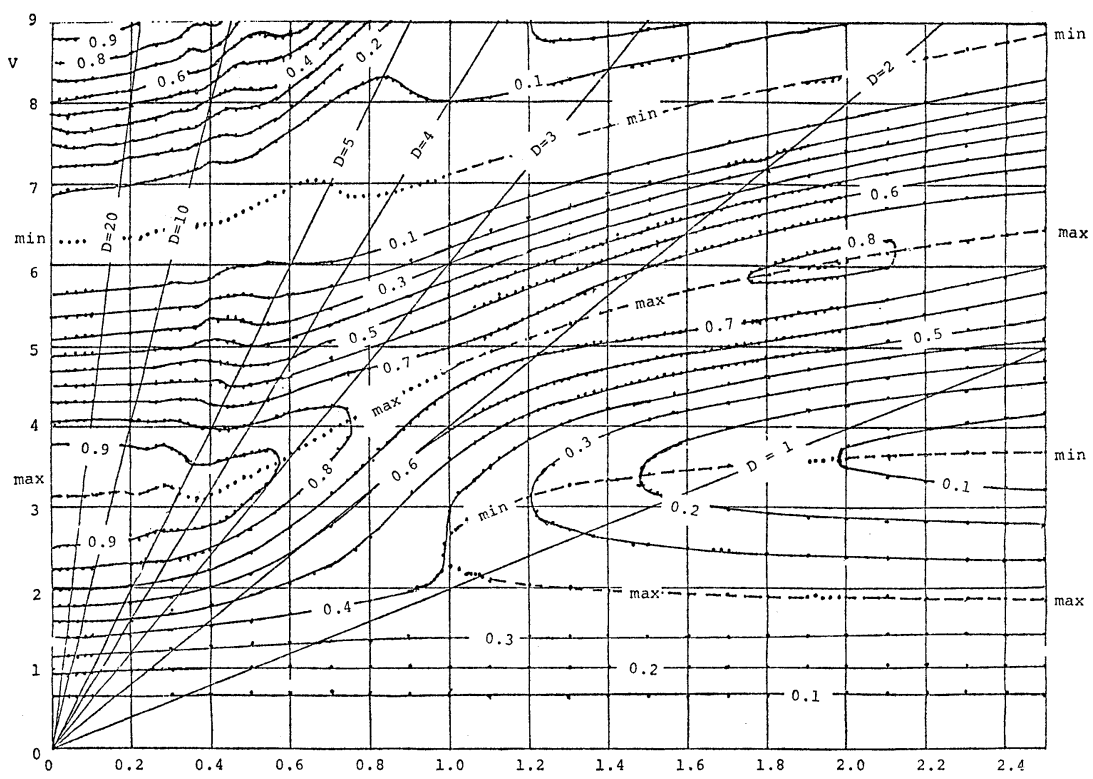

Fig. 9 Intensity of the 1st-order Brag maximum in the neighbourhood of $v \cong \pi$, and for small values of $\theta$.

complementary, the 0-order under 1st-order Bragg incidence becoming maximum at intermediate locations: $v=2 n \pi$ (cf. Fig. 3).

\subsection{Detailed Dependency of the 1st-Order Bragg} Maximum on the Parameters

The 1st-order Bragg maximum being of prime importance in practical applications, we have constructed a detailed $\theta v$-diagram based on a more detailed computation in the neighbourhood of the 1st-maximum $v=\pi$, as shown in Fig. 9. As is to be seen, the area of more than $90 \%$ light intensity is obtainable for $\theta<0.55$ and $v \sim \pi$. The maximum corresponds approximately to $v=\pi$ in the range $\theta \leqq$ 0.4 . For obtaining the maximum intensity for $\theta>$ $0.4, v$ must be increased somewhat along the dotted line-locus of the maximum. The ridge lies almost along the curve $\xi=$ const $(=4.3 \sim 4.5)$ and the 2 ndpeak near $\theta=2$ belongs to the mirror-type Bragg reflection rather than the true Bragg reflection, already.

Many approximate results are published in this region. Based on the Raman-Nath differencedifferential equations, Phariseau ${ }^{22)}$ gives the intensities under incidence angle $\phi$ in the neighbourhood of the Bragg angle for the high frequency limit $(D \gg 1)$ as

$$
\begin{aligned}
& I_{0}=\frac{1}{\sigma^{2}+\frac{1}{4}}\left[\sigma^{2}+\frac{1}{4} \cos ^{2}\left(\sigma^{2}+\frac{1}{4}\right)^{1 / 2} v\right], \\
& I_{1}=\frac{1}{4} \frac{\sin ^{2}\left(\sigma^{2}+\frac{1}{4}\right)^{1 / 2} v}{\sigma^{2}+\frac{1}{4}},
\end{aligned}
$$

where

$$
4 \sigma=\frac{1}{\theta}-2 \frac{\lambda}{\delta n \cdot \Lambda} \sin \phi .
$$

(cf. also Bhatia and Noble ${ }^{23)}$.)

If we write the incidence angle as $\sin \phi=p(\alpha / 2)$, we have $4 \sigma=(1-p) / \theta$. As we have $p=1$ at the Bragg incidence angle, we have

$$
I_{0}=\cos ^{2}(v / 2), \text { and } I_{1}=\sin ^{2}(v / 2) .
$$

under Bragg incidence. This is in agreement with our result in the limit $\theta=0$ (or $D=\infty$ ).

Klein $^{24)}$ obtained the light intensity along $v=\pi$ by the successive diffraction method and obtained $I_{1}=95 \%$ for $D \geqq 3.5$. The present result, on the other hand, indicates that $95 \%$ intensity is only obtainable for $\theta \leqq 0.3$ (or $D \geqq 5.3$ ), it being $I_{1}=93 \%$ at $D=3.5$. Notwithstanding this, Klein's curve for $\theta=\pi$ is comparatively in good agreement with our 
result, though that for maximum $I_{1}$ (along the ridge) appreciably deviates in regions of smaller $D$. (Klein gives $60 \%$ intensity for $D=2$, while the present result gives ca. $73 \%$.)

About a dozen papers have been published recently on the 1st-order Bragg diffraction of light by ultrasonic gratings or dielectric (thick hologram) gratings, in the cases of the Gaussian beams of light, bounded beams, cylindrical beams, 3-dimensional grating, not-sharply-bounded soundbeam, etc., either on the basis of the coupled-wave theory, or on the normal mode theory. Here we do not refer to them in detail because these are outside the scope of this paper. Moreover, all these calculations are performed in the range of extremely low values of $\theta(\ll 1)$, and mostly at a few discrete values of $v$ (cf. Moharam et al. ${ }^{24 a}$ ) and Konstantinov et al. ${ }^{24 \mathrm{~b})}$ for further references on these problems).

\section{INTENSITY DISTRIBUTION AMONGST ORDERS OF THE DIFFRACTION SPECTRUM}

We made calculations up to the 11th-order Bragg incidence at some discrete points of the $\theta v$-diagram. Figures 10 12 show some such examples. Clear and marked Bragg maxima are discernible in Fig. 11 , corresponding to $\theta=6.25$ and $D=0.7(v=8.75)$. In this case $1^{B}$-order (Bragg side 1st-order) spectrum becomes maximum under 1st-order Bragg incidence, $2^{B}$-order (Bragg side 2nd-order) spectrum becomes maximum under the 2nd-order Bragg incidence, and so on. Thus the Bragg maximum is apparent up to the 7th-order spectrum, the Bragg order being of the highest intensity -also higher than the 0-order-up to this incidence angle. Under the 8th-order Bragg incidence, however, the 8th-order spectrum is weaker than the 7thorder, though the 8th-order Bragg maximum is
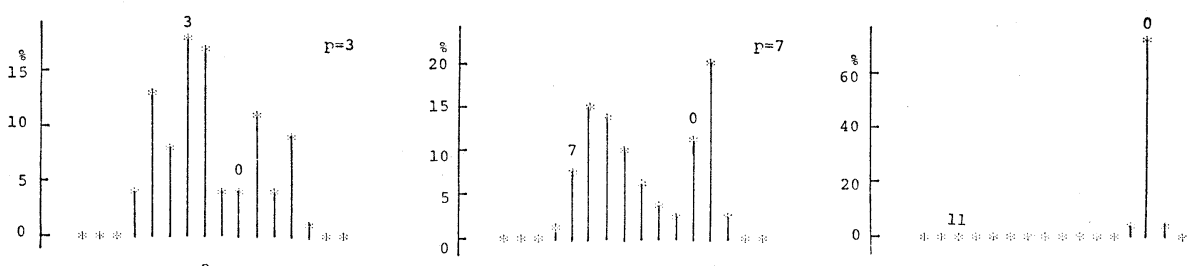

$p=11$
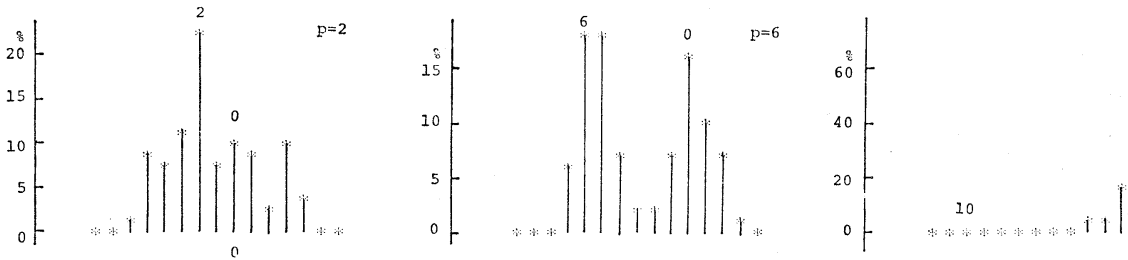

$p=10$
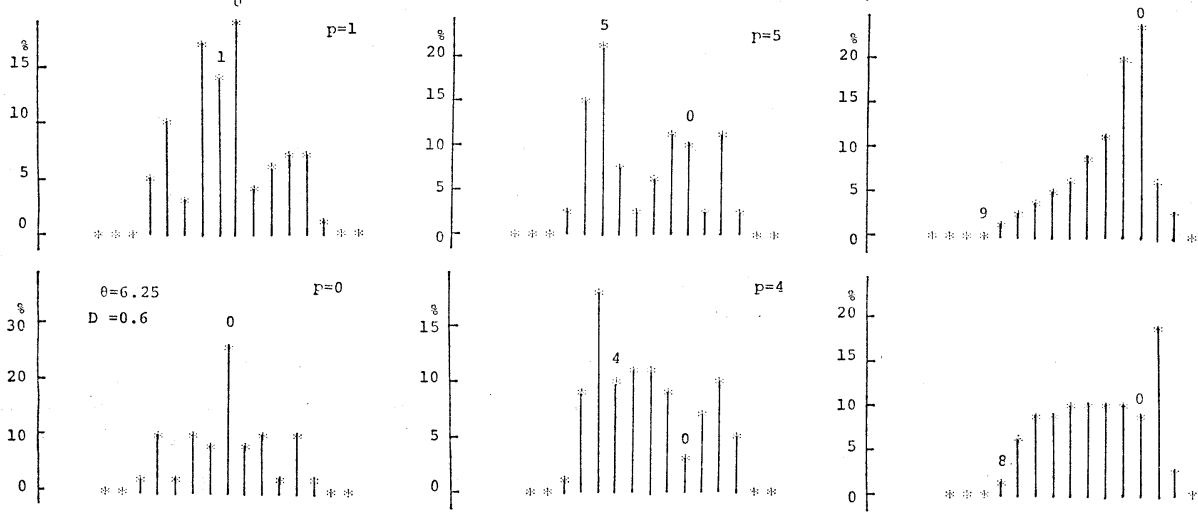

$\mathrm{P}=8$

Fig. 10 Intensity distribution amongst orders of the diffraction spectrum under various incidence angles from normal incidence $(p=0)$ to 11th-order Bragg incidence $(p=11)$ : $\theta=6.25, D=0.6$. 


\section{O. NOMOTO and A. OUCHI: DIFFRACTION OF LIGHT BY ULTRASOUND}
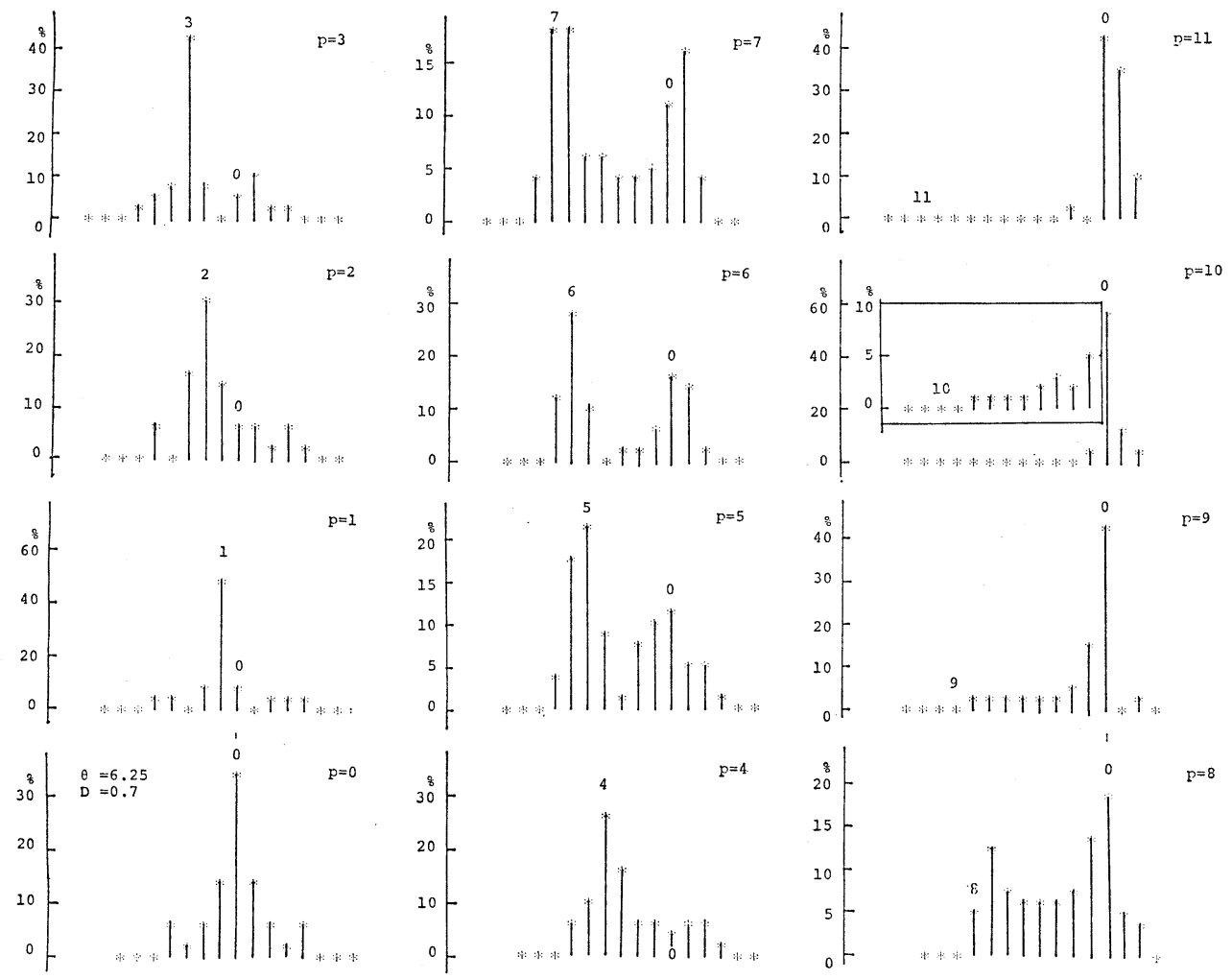

Fig. 11 Intensity distribution amongst orders of the diffraction spectrum under various incidence angles from normal incidence $(p=0)$ to 11th-order Bragg incidence $(p=11)$ : $\theta=6.25, D=0.7$.

still taking place because the $8^{B}$-order is stronger under the 8th-order Bragg angle than under both the 7th and the 9th-order Bragg incidence angles. No Bragg reflection is discernible for the orders $9^{B}, 10^{B}$, and $11^{B}$.

In case $\theta=6.25$ and $D=0.6(v=7.5)$ (Fig. 10), Bragg reflection is less marked. In this case $1^{B_{-}}$ order under Bragg incidence of this order is weaker than the $2^{B}$-order, and under Bragg incidence of the 4 th-order, the $4^{B}$-order (Bragg order) is weaker than the $5^{B}$-order. Under Bragg incidence of the 7th-order, the Bragg order (7th-order) is weaker than the $6^{B}, 5^{B}$, and $4^{B}$-orders. A detailed inspection of the figures, however, ascertains that all these cases correspond to Bragg maximum with respect to the incidence angle. On the other hand, Bragg reflection is not taking place for the $8^{B_{-}}$ order and for higher orders. Of some interest is the intensity distribution for the case $p=8$ (8th- order Bragg incidence). Here the intensities are sensibly the same for several orders $-0 \sim 6^{B}$. As these spectra are Doppler-shilfted each other with frequency intervals equal to the sound frequency, we have means of obtaining several light beams of mutually equally spaced frequencies and of sensibly the same intensity. This might be of use in some application purposes using light modulation by ultrasound. Similar circumstances are also to be met in cases corresponding to other values of the parameters.

Figure 12 shows the intensity distribution for $\theta=$ $6.25, D=1(v=12.5)$. In this case marked "Bragg minimum" is observed for the $1^{B}, 2^{B}, 3^{B}$ and $4^{B}-$ order spectra under respective Bragg incidence angles, while the orders $5^{B}, 6^{B}, 7^{B}$ and $8^{B}$ exhibit apparent Bragg maximum under respective Bragg angles. The $9^{B}$-order also exhibits Bragg maximum, though the intensity is weaker than the $8^{B}$-order 

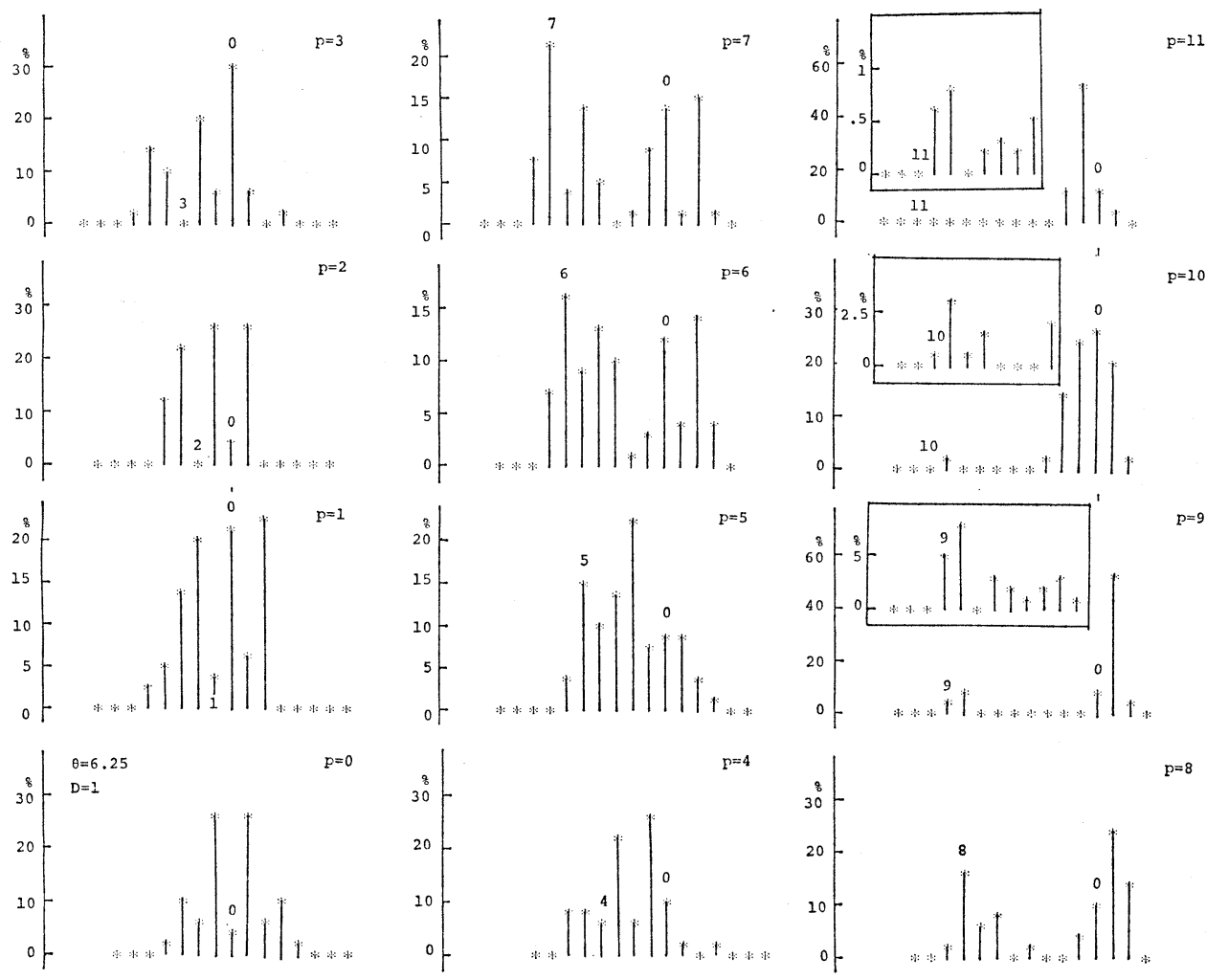

Fig. 12 Intensity distribution amongst orders of the diffraction spectrum under various incidence angles from normal incidence $(p=0)$ to 11 th-order Bragg incidence $(p=11)$ : $\theta=6.25, D=1.0$.

under 9th-order Bragg incidence.

Many other cases were calculated for various values of $\theta$ and $D$. Here the general tendency was the increase of the maximum Bragg order with increase in the value of $\theta$.

\section{RAY OPTICS INTERPRETATION}

The mechanism for the generation of the diffraction spectrum being rather complex in the present case of the diffraction by thick grating, any approximate interpretation based on some more intuitive mechanism might be of help in understanding the nature of the phenomenon. Phase-lattice approximation is approximately valid in the Raman-Nath region of Fig. 1, near the abscissa of the $\theta v$-diagram. True Bragg mechanism holds true in the region $\theta<1 / 2$, in the neighbourhood of the ordinate axis.

In the remaining rather large area of the $\theta v$ diagram, we propose that the approximate mech- anism pertaining to this phenomenon is the mirror type Bragg reflection.

Soon after the discovery of the phenomenon of the diffraction of light by ultrasound, Lucas and Biquard $^{33}$ traced the trajectories of the rays of light across the ultrasound beam in the case of the normal incidence of light. They obtained the result that the rays nearly focussed at distances $\xi=1.7$ and formed periodic intensity maxima (focal lines) with the sound wavelength as the period.

Ray optics theory proposed by Nomoto ${ }^{17)}$ is based on the assumption that the rays of light exiting the ultrasound under angles between

$$
\left.\begin{array}{c}
\left(n-\frac{1}{2}\right) \alpha<\phi<\left(n+\frac{1}{2}\right) \alpha, \\
(n=0, \pm 1, \pm 2, \ldots)
\end{array}\right\}
$$

contribute to the diffraction spectrum of the $n$-thorder. Here the angle $\phi$ is measured with respect 


\section{O. NOMOTO and A. OUCHI: DIFFRACTION OF LIGHT BY ULTRASOUND}

Table 4 Trajectories of the rays of light across the ultrasound beam.

Abbreviations: $\quad k_{0}{ }^{2}=k_{i}{ }^{2}+k_{g}{ }^{2}, k_{i}=\sin \alpha_{i}, k_{g}=\frac{g}{2 \sqrt{\mu / n_{0}}}, \alpha_{i}=\frac{\pi z_{i}}{\Lambda}, \alpha=\frac{\pi z}{\Lambda} ; I_{\gamma i}^{\gamma}(k)=\int_{\gamma i}^{\gamma} \frac{d \gamma}{\sqrt{1-k^{2} \sin ^{2} \gamma}},\left(\gamma>\gamma_{i}\right)$

\begin{tabular}{|c|c|c|c|c|c|}
\hline$k_{0}$ & $\begin{array}{l}\text { Non-oscillating } \\
\text { beam } k_{0}>1\end{array}$ & $\begin{array}{l}\text { Marginal ray } \\
k_{0}=1\end{array}$ & $\begin{array}{l}\text { Oscillating beam } \\
k_{0}<1\end{array}$ & & \\
\hline$\alpha_{i}$ & $\alpha_{i}^{\mathrm{C}}<\alpha_{i} \leqq 90^{\circ}$ & $\cos \alpha_{i}^{\mathrm{C}}=k_{g}$ & $\begin{array}{l}\text { Central ray } \\
\alpha_{i}=0\end{array}$ & $\begin{array}{l}\text { Non-central ray } \\
0<\alpha_{i}<\alpha_{i} \mathrm{C}\end{array}$ & $\begin{array}{l}\text { Normal incidence } \\
(g=0)\end{array}$ \\
\hline$k$ & $k_{0}^{\prime}=\frac{1}{k_{0}}<1$ & $\begin{array}{l}k_{0}=1 \\
k_{i}=\sin \alpha_{i}{ }^{\mathrm{C}} \\
k_{g}=\cos \alpha_{i}{ }^{\mathrm{C}}\end{array}$ & $\begin{aligned} k_{i} & =0 \\
k_{0} & =k_{g}=\cos \alpha_{i}{ }^{\mathrm{c}} \\
& <1\end{aligned}$ & $k_{g}<k_{0}<1$ & $\begin{array}{l}k_{g}=0 \\
k_{0}=k_{i}<1\end{array}$ \\
\hline$\xi *$ & $\begin{array}{l}k_{0}^{\prime} I_{r i}^{r}\left(k_{0}^{\prime}\right) \\
=k_{0}^{\prime}\left[K\left(k_{0}^{\prime}, \alpha\right)\right. \\
\left.\quad-K\left(k_{0}^{\prime}, \alpha_{i}\right)\right]\end{array}$ & $\begin{aligned} \frac{1}{2} & {\left[\log \frac{1+u}{1-u}\right.} \\
& \left.\quad-\log \frac{1+k_{i}}{1-k_{i}}\right] \\
u= & \sin \alpha\end{aligned}$ & $\begin{array}{l}I_{0}^{\gamma}\left(k_{0}\right) \\
=K\left(k_{g}, \gamma\right)\end{array}$ & $\begin{array}{l}I_{\gamma i}^{\gamma}\left(k_{0}\right) \\
=K\left(k_{0}, \gamma\right) \\
\quad-K\left(k_{0}, \gamma_{i}\right)\end{array}$ & $\begin{array}{l}I_{\pi / 2}^{\gamma}\left(k_{i}\right) \\
=K-K\left(k_{i},(\pi-\gamma)\right)\end{array}$ \\
\hline$\alpha$ & $\begin{aligned} & \alpha_{i}=\gamma_{i}, \\
& \alpha=\gamma,\left(\gamma \leqq 90^{\circ}\right) \\
& \alpha=\gamma-180^{\circ}, \\
&\left(90^{\circ}<\gamma \leqq 180^{\circ}\right)\end{aligned}$ & $\begin{array}{c}u= \pm \sin \alpha \frac{1-\mathrm{ce}^{-2 \xi}}{1+\mathrm{ce}^{-2 \xi}} \\
c=\frac{1-k_{i}}{1+k_{i}}\end{array}$ & $\sin \alpha=k_{g} \sin \gamma$ & $\begin{array}{l}\sin \gamma_{i}=\frac{k_{i}}{k_{0}} \\
\sin \alpha=k_{0} \sin \gamma\end{array}$ & $\sin \alpha=k_{i} \sin \gamma$ \\
\hline$\gamma$ & $\begin{aligned} \sin \gamma= & \operatorname{sn}\left[K\left(k_{0}^{\prime}, \gamma\right.\right. \\
& \left.+\frac{\xi}{k_{0}^{\prime}}\right]\end{aligned}$ & & $\sin \gamma=\operatorname{sn} \xi$ & $\begin{array}{l}\sin \gamma \\
=\operatorname{sn}\left[K\left(k_{0}, \gamma_{i}\right)\right. \\
\quad+\xi]\end{array}$ & $\sin \gamma+\operatorname{sn}(K+\xi)$ \\
\hline$s$ & $2 \sqrt{k_{0}^{2}-\sin ^{2} \alpha}$ & $2 \cos \alpha$ & $2 \sqrt{k_{g^{2}}-\sin ^{2} \alpha}$ & $2 \sqrt{k_{0}^{2}-\sin ^{2} \alpha}$ & $2 \sqrt{k_{i}{ }^{2}-\sin ^{2} \alpha}$ \\
\hline
\end{tabular}

* The initial part only of the $\xi$-curve is indicated in elliptic integrals. The remaining part is easily to be obtained by taking into acount the periodicity-with period $4 K$-of the elliptic functions.

to the incident light. The original derivation ${ }^{17}$ being somewhat cumbersome, we outline here the essential parts of the revised derivation in a simplified form, with main emphasis on the treatment of the Bragg reflection. The results as regards the trajectories of the rays of light are summarized in Table 4.

The trajectories of the rays of light across the ultrasound beam with distribution of the refractive index

$$
n=n_{0}+\mu \cos \frac{2 \pi z}{\Lambda}
$$

is obtainable from the Snell's law

$$
\frac{1}{n}\left[1+\left(\frac{d z}{d x}\right)^{2}\right]^{1 / 2}=\frac{1}{n_{i}}\left[1+g^{2}\right]^{1 / 2} ; g=\left(\frac{d z}{d x}\right)_{i} \text {. }
$$

Here $g$ means the slope of the incident light with respect to the sound wavefront. As $\left(\mu / n_{0}\right) \ll 1$, we have from (5.2) and (5.3) the relation:

$$
\begin{aligned}
\frac{d z}{d x} & =\left[\frac{2}{n_{0}}\left(\cos 2 \alpha-\cos 2 \alpha_{i}\right)+g^{2}\right]^{1 / 2} \\
& =\left[\frac{4}{n_{0}}\left(\sin ^{2} \alpha_{i}-\sin ^{2} \alpha\right)+g^{2}\right]^{1 / 2},
\end{aligned}
$$

where

$$
\alpha=\frac{\pi z}{\Lambda}, \quad \text { and } \quad \alpha_{i}=\frac{\pi z_{i}}{\Lambda}
$$

and $z_{i}$ means the initial ordinate of the ray of light at the plane of incidence into sound beam. If we have $(d z / d x)=0$ at some value of $z=z_{0}$, this corresponds to the maximum (or minimum) of $z$. The maximum is determined by

$$
\sin \frac{\pi z_{0}}{\Lambda}=\sin ^{2} \alpha_{i}+{k_{g}}^{2}=k_{i}{ }^{2}+k_{g}{ }^{2}=k_{0}{ }^{2}
$$


where

$$
k_{g}=\frac{g}{2 \sqrt{\mu / n_{0}}}, \text { and } k_{i}=\sin \alpha_{i},
$$

while the minimum is $-z_{0}$.

In this case the ray oscillates between $z_{0}$ and $-z_{0}$ within the width of an ultrasound wavelength. The maximum initial ordinate $z_{i}^{\mathrm{C}}$ corresponds to $z_{0}=\Lambda / 2$, and this determines the half-width of the oscillating beam by $\cos ^{2} \alpha_{i}=k_{g}{ }^{2}$, or

$$
\cos \left(\pi z_{i} \mathrm{C} / \Lambda\right)=q / 4=m / 4 \sqrt{\theta} .
$$

Here $q$ is the initial slope parameter related to the incidence Bragg order $m$ by $q=m / \sqrt{\bar{\theta}}$ ( $m$ is a continuous variable and not necessarily an integer in case of the ray optics theory). The trajectories are expressible in terms of the elliptic integral of the first kind $K(k, \gamma)$ and the complete elliptic integral $K$,

$$
K(k, \gamma)=\int_{0}^{r} \frac{d \gamma}{\sqrt{1-k^{2} \sin ^{2} \gamma}} ; \quad K=K\left(k, \frac{\pi}{2}\right)
$$

with suitable parameter depending on various cases (cf. Table 4). The intensity of the diffraction spectrum is obtainable by enumerating the number of (equidistant) incident rays exiting the sound- beam under angles within definite range (cf. (5.1)). The Table indicates also the slope

$$
s=\frac{p}{\sqrt{\theta}} \frac{1}{\sqrt{\mu / n_{0}}} \frac{d z}{d x}
$$

Figure 13 shows the trajectories of the rays of light in case of the oblique incidence corresponding to $\alpha_{i}{ }^{\mathrm{C}}=60^{\circ}$. Detailed results of this kind were obtained before, ${ }^{17)}$ but the range of computation here is widely extended-as regards the parameter $\xi$ and also for the angle of incidence-for the purpose of comparison with the normal mode theory.

Oscillating beam only is depicted in Fig. 13 because the non-oscillating beam does not contribute to the Bragg order spectrum. A salient feature of the oscillating beam is that they form nearly parallel bundles at nearly periodic distances and in directions nearly mirror-reflected to and fro within the width of a sound wavelength. By constructing figures of this kind under various values of $\alpha_{i}{ }^{\mathrm{c}}$, we see that the periodic distance becomes somewhat longer with decrease of $\alpha_{i}{ }^{\mathrm{c}}$, i.e., with increase in the angle of incidence. In the neighbourhood of $\xi \cong 3.5,10$ and 17 in Fig. 13 we see the nearly parallel beams in mirror-reflected directions with respect to the incident beam, and

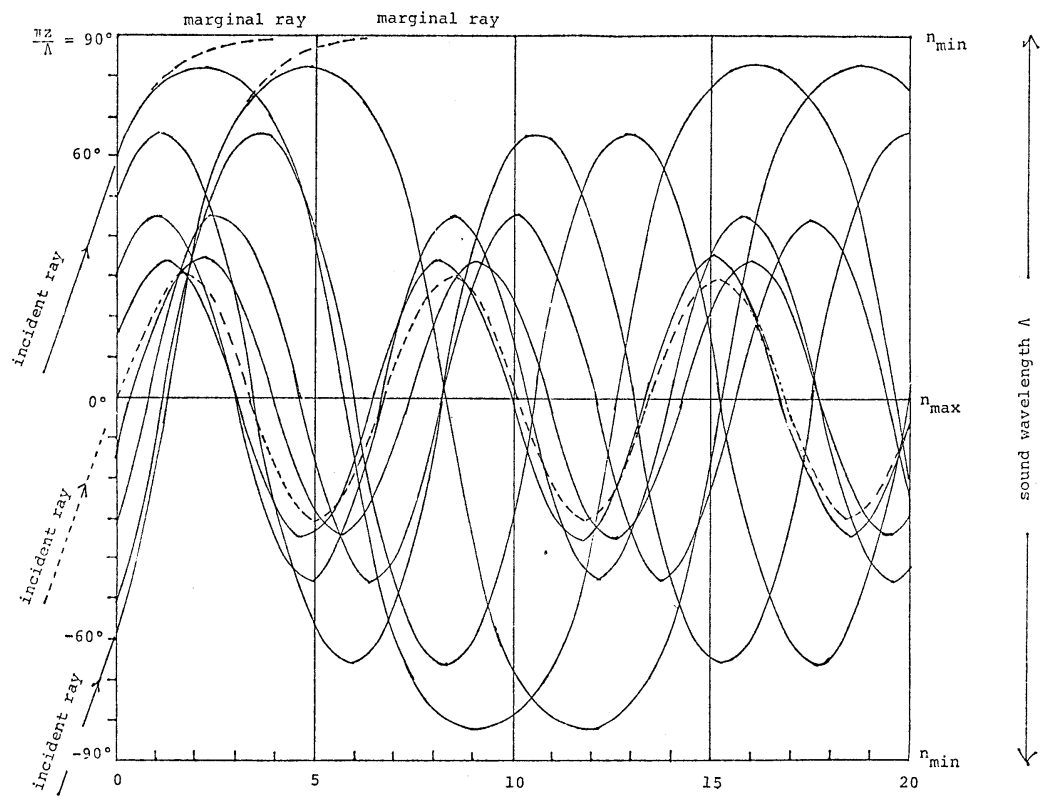

Fig. 13 Trajectories of the rays of light across the ultrasound beam: oblique incidence, $\alpha_{i}{ }^{\mathrm{C}}=60^{\circ}$ (width of the oscillating beam $=2 / 3$ the width of the incident beam). 

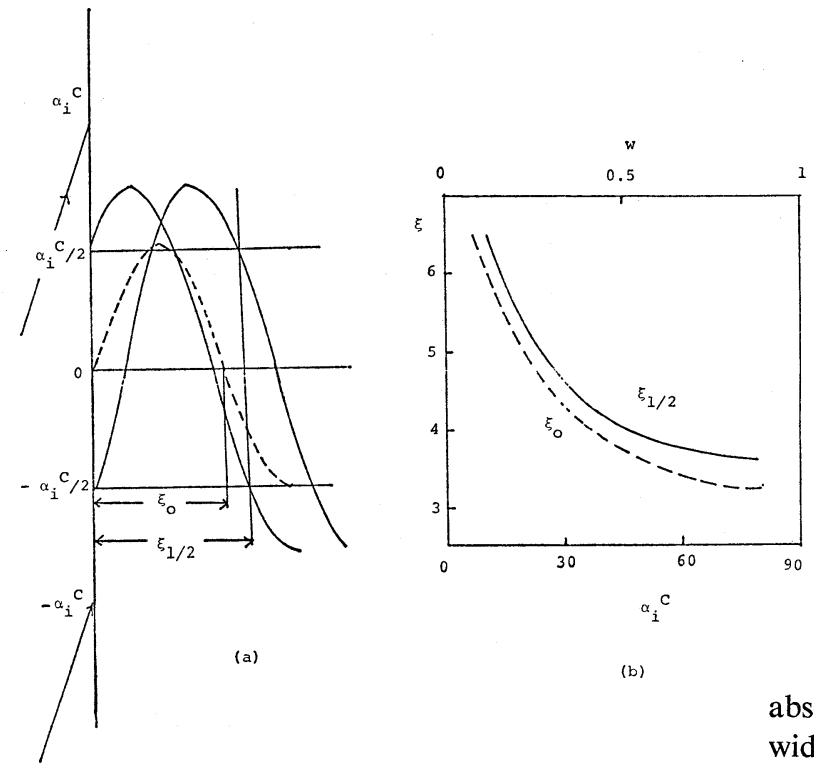

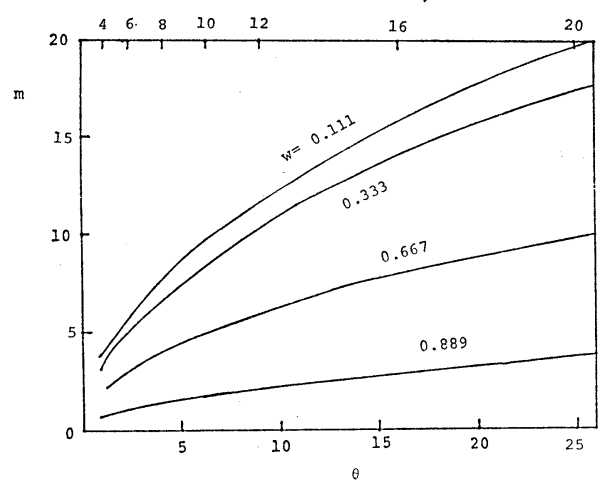

Fig. 15 Relation between incidence Bragg order $m$ and $\theta$ with $w$ as parameter.
Fig. 14 Recurrence periods $\xi_{0}$ and $\xi_{1 / 2}$ and their dependence on $\alpha_{i}{ }^{\mathrm{C}}$ and $w$, width of the oscillating beam.

these contribute to the Bragg reflection.

Figure 14(a) illustrates the recurrence periods $\xi_{0}$ and $\xi_{1 / 2}$ and (b) shows their dependence on $\alpha_{i}{ }^{\mathrm{c}}$. Here $\xi_{0}$ is the first recurrence period of the central ray and corresponds to twice the value of the complete elliptic integral with argument $k_{g}$, i.e.,

$$
\xi_{0}=2 K\left(k_{g}, \pi / 2\right) \text {. }
$$

$\xi_{1 / 2}$ corresponds to the distance at which the rays incident with initial ordinates $\alpha_{i}= \pm \alpha_{i}{ }^{\mathrm{C}} / 2$, i.e., the rays indicating the boundary of the halfwidth of the oscillating beam attain the points $\alpha=\mp \alpha_{i}{ }^{\mathrm{C}} / 2=$ $-\alpha_{i}$, respectively. At this distance the two rays are completely parallel and also in phase, and in the direction mirror-reflected to the incident rays. Thus the distance $\xi_{1 / 2}$ approximately indicates the optimum value of $\xi$ for obtaining the Bragg reflection of the corresponding order $m$. This corresponds to twice the complete elliptic integral with argument $k_{0}\left(\alpha_{i}^{\mathrm{C}} / 2\right)$, i.e.,

$$
\xi_{1 / 2}=2 K\left[k_{0}\left(\alpha_{i}^{\mathrm{C}} / 2\right), \pi / 2\right]
$$

(The curves in Fig. 13 and the values $\xi_{0}, \xi_{1 / 2}$ were calculated by numerical integration by the Simpson method, and partly checked by interpolation from tabular values (cf. Belyakov et $a l .{ }^{25}$ ). The upper abscissa in Fig. 14(b) indicates $w\left(=\alpha_{i}^{\mathrm{C}} / 90^{\circ}\right)$, the width of the oscillating beam.

Figure 15 indicates the relation between the incident Bragg order $m$ and $\theta$, with the width of the oscillating beam $w$ as parameter. The upper abscissa $v$ corresponds to the assumption that $\xi=$ 4, so that we have $v=\xi \sqrt{\theta}=4 \sqrt{\theta}$. More reasonable value of $v$ is obtainable by using $\xi_{1 / 2}$ corresponding to each value of $w$ from Fig. 14.

The ray optics result is in comparatively good agreement with the above-mentioned exact calculations. The existence of the Bragg maximum as well as the Bragg minimum according to the value of the parameter $\xi$ is self-explanatory from the behaviour of the oscillating beam. In more detail, we see firstly that the locations of Bragg maxima for the 1st, 2nd, 3rd and 4th-order spectra as indicated in Fig. 1 is in agreement with the ray optics result. Namely the 1st Bragg maximum corresponding to the lowest value of $v$ nearly located along the curve $\xi=4$, and the next Bragg maximum located nearly along $\xi=10 \sim 12$ are understandable from Fig. 13.

Secondary, the suitable value of $\xi$ for obtaining Bragg reflection becomes higher for higher Bragg order $m$, because both $\xi_{1 / 2}$ and $m$ increases with decrease in $w$ (cf. Fig. 14(b) and Fig. 15). This is in agreement with the exact result as obtained in 4. We refer especially to Fig. 12, corresponding to $\theta$ $=6.25$ and $D=1.0$, i.e., $v=12.5$ and $\xi=5.0$. While the lower orders, 1st 4th, showed "Bragg minimum" in this case, the higher orders, 5th $\sim 9$ th, exhibited Bragg maximum. On the other hand we see in Fig. 
11 , corresponding to $\theta=6.25$ and $D=0.7$, i.e., $v=$ 8.75 and $\xi=3.5$, that the orders 1st to 8th show Bragg maximum, while the 9 th-order does not. A more detailed inspection reveals that each Bragg order up to the 6th is higher for $\xi=3.5$ (Fig. 11) than for $\xi=5.0$ (Fig. 12). The 7th 9th Bragg orders are higher in case $\xi=5.0$ than in case $\xi=3.5$. This indicates that the suitable value of $\xi$ becomes higher for higher Bragg orders under constant value of $\theta$, in accordance with the ray-optics result.

Thirdly we may infer from Fig. 15 that higher order Bragg maximum as for example the 20thorder may occur only for sufficiently large values of $\theta$, i.e., $\theta \geqq 25$. This is outside the range of our exact calculations by the normal mode theory, but we expect that the prediction of the ray optics theory may be of help in obtaining a wide prospect of the phenomenon, if not so exact from quantitative point of view.

Systematic experiments on the higher order Bragg diffraction is performed by Nomoto. ${ }^{26)}$ One of these experiments, corresponding to $\theta=6.78$, $\xi=3.5(v=9.11)$ is explained by the ray optics theory $\left(\right.$ Nomoto $\left.^{17)}\right)$. Bragg maxima up to the 9thorder was observed in this experiment. Ray optics calculation obtained the intensities of the 1st-order Bragg maximum as $50 \%$, and that of the 5 thorder Bragg maximum as $30 \%$, while the experimental values were $46 \%$ and $20 \%$, respectively. Another experiment performed at $D=0.4$ gave Bragg maxima up the 22nd-order. Quantitative intensity measurements were not made in this case, but we can infer that $\xi \geqq 3.5$ because very selective Bragg maxima are obtained. If $\xi=3.5$, we have $v=13.4$ and $\theta=19.14$, if $\xi=4$, we have $v=20$, and $\theta=25$, and if $\xi=4.5$, we have $v=25.31$ and $\theta=$ 31.64 .

\section{REFERENCES}

1) L. Brillouin, "Diffusion de la lumière et des rayons $\mathrm{X}$ par un corps transparent homogène; influence de l'agitation thermiques," Annale de Physique Paris 17, 88-122 (1922).

2) P. Debye and F. W. Sears, "On the scattering of light by supersonic waves," Proc. Nat. Acad. Sci. 18, 409-414 (1932).

3) R. Lucas et P. Biquard, "Propriétés optiques des milieux solides et liquides soumis aux vibrations élastiques ultra sonores," J. de Physique 3, 464-474 (1932).

4) C. V. Raman and N. S. Nagendra Nath, "The diffraction of light by high-frequency sound waves: Part I, Part II, Part III. Doppler effect and coherence phenomena," Proc. Indian Acad. Sci. 2A, 406-412, 413-420 (1935); 3A, 75-84 (1936).

5) L. Brillouin, Diffraction de la Lumière par des Ultra-Sons (Actualités Scientifiques et Industrielles No. 59, Hermann, Paris, 1933), p. 32.

6) C. V. Raman and N. S. Nagendra Nath, "The diffraction of light by high frequency sound waves: Part IV. Generalized theory; Part V. General considerations-oblique incidence and amplitude changes," Proc. Indian Acad. Sci. 3A, 119-125; 459-465 (1936).

7) N. S. Nagendra Nath, "The diffraction of light by high frequency sound waves: Generalized theory. The asymmetry of the diffraction phenomena at oblique incidence," Proc. Indian Acad. Sci. 4A, 222-242 (1936).

8) R. Extermann et G. Wannier, "Théorie de la diffraction de la lumière par les ultrasons," Helv. Phys. Acta 9, 520-532 (1936).

8a) T. Yamamoto, "The Rayleigh-Ritz and WeinsteinBazley methods applied to a class of ordinary differential equations of the second order. I, II," Math. Japon. 19, 95-111 (1974); SIAM J. Numer. Anal. 12, 428-438 (1975).

9) R. C. Extermann, "Théorie de la diffraction de la lumière par les ultrasons. II," Helv. Phys. Acta 10, 185-217 (1937).

10) E. H. Wagner, "Zur Beugung paralleler Lichtbundel an laufenden Ultraschallwellen. I, II, III, IV," Zeits. Phys. 141, 604-621, 622-642 (1955); 142, 249-256, 412-430 (1955).

10a) E. H. Wagner, "Zur Braggschen Reflexion von Licht an Ultraschallwellen," Acustica 6, 17-24 (1956).

11) G. Plancke-Schuyten and R. Mertens, "The diffraction of light by progressive supersonic waves. Oblique incidence of light. II. Exact solution of the Raman-Nath equations," Physica 62, 600-613 (1972).

12) T. Tamir and H. C. Wang, "Scattering of electromagnetic waves by a sinusoidally stratified halfspace. I. Formal solution and analytic approximations. II. Diffraction aspects at the Rayleigh and Bragg wavelengths," Canad. J. Phys. 44, 2073-2094, 2461-2494 (1966).

13) R. S. Chu and T. Tamir, "Guided-wave theory of light diffraction by acoustic microwaves," IEEE Trans. Microwave Theor. and Techn. MTT-17, 1002-1020 (1969); MTT-18, 486-504 (1970).

14) O. Nomoto, "Diffraction of light by ultrasound: Extension of the Brillouin theory," Jap. J. Appl. Phys. 10, 611-620 (1971). 


\section{O. NOMOTO and A. OUCHI: DIFFRACTION OF LIGHT BY ULTRASOUND}

15) O. Nomoto, "Diffraction de la lumière par les ultrasons: Calcul des intensités spectrales en cas des incidences de Bragg de divers orderes selon la théorie de Brillouin," J. de physique, Colloque C6, C6-189-195 (1972).

16) O. Nomoto, "Bragg-diffraction of light by ultrasound: Extended Brillouin theory," Memoirs Defense Academy 15, 121-149 (1975).

17) O. Nomoto, "Geometrical optical theory of the diffraction of light by ultrasonic waves. Approximate treatment. I, II," Bull. Kobayasi Inst. Phys. Res. 1, 42-71, 189-220 (1951).

18) J. Pouliquen et N. Segard, "Diffraction a l'infini d'une onde lumineuse orthogonale a une onde ultrasonore plane et progressive. Calcul de l'intensite des raies du spectre par ordinateur electronique," Revue d'Optique 42, 559-583, 616-645 (1963).

19) N. W. McLachlan, "Theory and Application of Mathieu Functions" (Oxford Univ. Press, 1947, Dover, New York, 1964).

20) G. W. Willard, "Criteria for normal and abnormal ultrasonic light diffraction effects," J. Acoust. Soc. Amer. 21, 101-108 (1949).

21) L. C. Bark, N. Z. Dmirtieva, L. N. Zakhar'ev, and A. A. Lemanskii, Tables of Characteristic Values of the Mathieu Equation (in Russian) (Computation Center, Akad. Nauk USSR, Moskva, 1970).
22) P. Phariseau, "On the diffraction of light by progressive supersonic waves. Oblique incidence: Intensities in the neighbourhood of the Bragg angle," Proc. Indian Acad. Sci. A44, 165-170 (1956).

23) A. B. Bhatia and W. J. Noble, "Diffraction of light by ultrasonic waves. I. General theory, II. Approximate expressions for the intensities and comparison with experiment," Proc. Roy. Soc. A220, 356-368, 369-385 (1953).

24) W. R. Klein, "Theoretical efficiencies of Bragg devices," Proc. IEEE 54, 803-804 (1966).

24a) M. G. Moharam, T. K. Gaylord, and R. Magnusson, "Bragg diffraction of finite beams by thick gratings," J. Opt Soc. Amer. 70, 300-304 (1980).

24b) O. V. Konstantinov, M. M. Panakhov, and Yu. F. Romanov, "Electro-dynamic perturbation theory for light diffraction from 3-D gratings." Opt. Spektrosk. 46, 979-985 (1979) [Opt. Spectrosc. (USSR) 46, 551-554 (1979)].

25) V. M. Belyakov, R. I. Kravsova, and M. G. Rappoport, Tables of Elliptic Integrals, Vol. 1 (1962), Vol. 2 (1963) (Akad. Nauk USSR (in Russian)).

26) O. Nomoto, "Studien über die Beugung von Licht an Ultraschallwellen. II. Beugung von Licht an Ultraschallwellen bei schiefem Einfall des Lichtes auf die Schallwellenfront. Braggsche Reflexion," Proc. Phys-Math. Soc. Jpn 24, 613-639 (1942). 